\title{
Enhanced gauge symmetry and winding modes in double field theory
}

\author{
G. Aldazabal, ${ }^{a, b}$ M. Graña, ${ }^{c}$ S. Iguri, ${ }^{d}$ M. Mayo, ${ }^{a, b}$ C. Nuñez ${ }^{d, e}$ and J.A. Rosabal ${ }^{e}$ \\ ${ }^{a}$ Centro Atómico Bariloche, \\ 8400 S.C. de Bariloche, Argentina \\ ${ }^{b}$ Instituto Balseiro (CNEA-UNC) and CONICET, \\ 8400 S.C. de Bariloche, Argentina \\ ${ }^{c}$ Institut de Physique Théorique, CEA/Saclay, \\ 91191 Gif-sur-Yvette Cedex, France \\ ${ }^{d}$ Instituto de Astronomía y Física del Espacio (CONICET-UBA), Universidad de Buenos Aires, \\ 1428 Buenos Aires, Argentina \\ e Departamento de Física, FCEN, Universidad de Buenos Aires, \\ C.C. 67 - Suc. 28, 1428 Buenos Aires, Argentina \\ E-mail: aldazabal@cab.cnea.gov.ar, mariana.grana@cea.fr, \\ siguri@iafe.uba.ar, martinmayo1991@gmail.com, carmen@iafe.uba.ar, \\ arosabal@df.uba.ar
}

ABSTRACT: We provide an explicit example of how the string winding modes can be incorporated in double field theory. Our guiding case is the closed bosonic string compactified on a circle of radius close to the self-dual point, where some modes with non-zero winding or discrete momentum number become massless and enhance the $\mathrm{U}(1) \times \mathrm{U}(1)$ symmetry to $\mathrm{SU}(2) \times \mathrm{SU}(2)$. We compute three-point string scattering amplitudes of massless and slightly massive states, and extract the corresponding effective low energy gauge field theory. The enhanced gauge symmetry at the self-dual point and the Higgs-like mechanism arising when changing the compactification radius are examined in detail. The extra massless fields associated to the enhancement are incorporated into a generalized frame with $\frac{O(d+3, d+3)}{O(d+3) \times O(d+3)}$ structure, where $d$ is the number of non-compact dimensions. We devise a consistent double field theory action that reproduces the low energy string effective action with enhanced gauge symmetry. The construction requires a truly non-geometric frame which explicitly depends on both the compact coordinate along the circle and its dual.

Keywords: String Duality, Bosonic Strings

ARXIV EPRINT: 1510.07644 


\section{Contents}

1 Introduction 1

2 Bosonic string compactified on a circle 3

2.1 Massless states and vertex operators at the self-dual radius 3

$\begin{array}{lll}2.2 & \text { Effective action at the self-dual radius and Higgs mechanism } & 6\end{array}$

3 Away from the self-dual point $\quad 7$

3.1 Vertex operators and amplitudes for $R \neq \tilde{R} \quad 7$

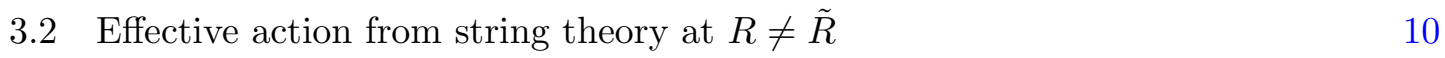

$\begin{array}{lll}3.3 & \text { Interpretation as a Higgsing } & 14\end{array}$

4 DFT and enhanced gauge symmetries $\quad \mathbf{1 5}$

$\begin{array}{lll}4.1 & \text { Some notions on DFT } & 16\end{array}$

$\begin{array}{lll}4.2 & \text { Circle reduction } & 19\end{array}$

$\begin{array}{lll}4.3 & \text { Extension and enhanced gauge symmetry } & 22\end{array}$

$\begin{array}{lll}4.4 & \text { Internal double space } & 23\end{array}$

$\begin{array}{ll}\text { 4.5 Effective action from DFT } & 26\end{array}$

5 Conclusions $\quad 28$

$\begin{array}{ll}\text { A Three-point amplitudes } & 30\end{array}$

\section{Introduction}

The frameworks of Generalized Complex Geometry (GCG) $[1,2]$ and Double Field Theory (DFT) [3-5] have been proposed in order to incorporate T-duality as a geometric symmetry. T-duality is a distinct symmetry of string theory associated to the fact that, being an extended object, a string can wrap cycles of the compact space, leading to the so-called winding states. These states are created by vertex operators involving both coordinates associated with momentum excitations and dual coordinates associated with winding excitations. Perturbations of the vacuum by these operators may lead to non-geometric backgrounds which correspond to field theories with interactions depending on both sets of coordinates.

The idea to describe these properties within a field theory in which the fields depend on the double set of coordinates has a long history [6-9], and it is the subject of current active research (see the recent reviews [10-12]). Generically these field theories must satisfy consistency constraints. A solution to these constraints is the so called section condition, which effectively leads to the elimination of half of the coordinates. Even if the original motivation is lost when choosing this solution and DFT shares the basic features of GCG in 
this case (both frames are based on an ordinary, undoubled, manifold), it provides an interesting tool for understanding underlying symmetries of the theory and some genuine stringy features like $\alpha^{\prime}$ corrections have been recently incorporated [13-17] in these formulations.

Alternative solutions to the constraint equations are the generalized ScherkSchwarz [18] like compactifications of DFT [19, 20]. These compactifications contain the generic gaugings of gauged supergravity theories, allowing for a geometric interpretation of all of them. In this framework, the doubled coordinates enter in a very particular way through the twist matrix which gives rise to the constant gaugings.

So far the DFT construction has considered only the massless states of the uncompactified string. The purpose of the present work is to provide an explicit example of how the string winding modes can be incorporated in the double field theory formulation. In particular, we show that the striking stringy feature of gauge symmetry enhancement at certain points in the compactification space can be accounted for in this context. We address this issue in the simplest example of closed bosonic string theory compactified on a circle of radius close to the self-dual point.

In order to pursue this construction we first recall some basic information of string compactification on a circle, including the enhancement of the gauge group at the self-dual point [21-24], with the aim of identifying the key ingredients to be incorporated in the DFT framework. We compute three-point scattering amplitudes in the bosonic string involving states with non-vanishing winding and compact momentum. The computation is performed at the self-dual point, with enhanced $\mathrm{SU}(2)_{L} \times \mathrm{SU}(2)_{R}$ gauge symmetry, but also slightly away from it, where some states acquire tiny masses and the gauge symmetry is spontaneously broken to $\mathrm{U}(1)_{L} \times \mathrm{U}(1)_{R}$. We then extract the effective field theory describing their interactions and study in detail the enhancement of the gauge symmetries and the Higgs-like mechanism that arises when changing the radius of the compact dimension. The string theory basics are presented in section 2 , and in section 3 we discuss the compactification away from the self-dual radius.

We then proceed to the construction of the Double Field Theory description in section 4. We start by presenting the usual Kaluza-Klein (KK) compactification of the metric and antisymmetric fields and then rewrite the results in a chiral basis, which is suitable to describe the extension to the left and right $\mathrm{SU}(2)$ gauge groups, as dictated by the string compactification. String computations as well as the identification of an $\frac{O(d+3, d+3)}{O(d+3) \times O(d+3)}$ structure, where $d$ is the number of non-compact spacetime dimensions, are used as a guide to build an extended frame that incorporates all the fields of the reduced theory and, in particular, the fields associated to the enhancement. The generalized frame formulation of DFT and its Scherk-Schwarz reduction are used to write down a consistent DFT effective action that coincides with the effective string field theory action with the enhanced symmetry.

Finally, we discuss the structure of the generalized frame. Interestingly enough, this frame must depend on both the coordinate on the circle and its dual, and it therefore furnishes a truly non-geometric construction. Concluding remarks and a brief outlook are presented in section 5 . 


\section{Bosonic string compactified on a circle}

In this section we introduce some basic concepts about the closed bosonic string compactified on a circle, which are needed throughout the rest of the paper.

\subsection{Massless states and vertex operators at the self-dual radius}

Consider the closed bosonic string compactified on a circle of radius $R$, with coordinate identification

$$
Y(\sigma, \tau)=y(\sigma+i \tau)+\bar{y}(\sigma-i \tau) \sim Y(\sigma, \tau)+2 \pi R,
$$

where $\sigma, \tau$ are the coordinates on the worldsheet. ${ }^{1}$

This periodicity has two effects. On the one hand, univaluedness of the wave function requires discrete momentum $p$ in the compact dimension, like in Kaluza Klein (KK) reduction in field theory. On the other hand, unlike point particles, strings can wind an integer number of times $\tilde{p}$ around the compact direction, i.e.

$$
Y(\sigma+2 \pi, \tau) \sim Y(\sigma, \tau)+2 \pi \tilde{p} R
$$

The dual coordinate $\tilde{Y}(\sigma, \tau)=y(\sigma+i \tau)-\bar{y}(\sigma-i \tau)$ satisfies

$$
\tilde{Y}(\sigma+2 \pi, \tau) \sim \tilde{Y}(\sigma, \tau)+2 \pi p \tilde{R}
$$

where we have defined the dual radius

$$
\tilde{R}=\frac{\alpha^{\prime}}{R}
$$

In the uncompactified theory, the massless fields are the metric, the antisymmetric tensor and the dilaton.

For one compact dimension, the metric and the B-field with one leg along the circle give rise to two massless KK U(1) gauge vectors while the metric with both legs along the circle gives rise to a massless scalar. Apart from these, at the self-dual radius

$$
R_{\mathrm{sd}}=\tilde{R}_{\mathrm{sd}}=\sqrt{\alpha^{\prime}}
$$

more vector states become massless and the $\mathrm{U}(1)_{L} \times \mathrm{U}(1)_{R}$ gauge group is enhanced to $\mathrm{SU}(2)_{L} \times \mathrm{SU}(2)_{R}$. Nine massless scalars, transforming in the $(3,3)$ representation, do also appear. This can be easily seen from the mass formula,

$$
M^{2}=-K^{2}=\frac{2}{\alpha^{\prime}}(N+\bar{N}-2)+\frac{k^{2}}{2}+\frac{\bar{k}^{2}}{2},
$$

and the level matching constraint,

$$
\bar{N}-N=p \tilde{p},
$$

\footnotetext{
${ }^{1}$ We use a bar to indicate right-moving quantities $(\bar{y}, \bar{N}, \bar{k}, \ldots$ are the right-moving coordinate, rightmoving oscillation number, right-moving momentum, etc). Not to be confused with complex conjugation.
} 
where $N=N_{x}+N_{y}\left(\bar{N}=\bar{N}_{x}+\bar{N}_{y}\right)$ is the left (right) moving number operator, involving the sum of the number operator along the circle $N_{y}\left(\bar{N}_{y}\right)$ and the number operator for the non-compact spacetime directions, denoted by $N_{x}\left(\bar{N}_{x}\right)$. The left- and right-momenta for the periodic dimension are

$$
k=\frac{p}{R}+\frac{\tilde{p}}{\tilde{R}}, \quad \bar{k}=\frac{p}{R}-\frac{\tilde{p}}{\tilde{R}} .
$$

For later convenience we introduce the following parameters with mass dimension

$$
\begin{aligned}
& m_{-}=R^{-1}-\tilde{R}^{-1}=\frac{1}{\alpha^{\prime}}(\tilde{R}-R), \\
& m_{+}=R^{-1}+\tilde{R}^{-1}=\frac{1}{\alpha^{\prime}}(\tilde{R}+R) .
\end{aligned}
$$

The massless states appearing at the self-dual radius are quoted below. ${ }^{2}$

1. The three vector states generating $\mathrm{SU}(2)_{L}$ have $\bar{N}_{x}=1, N_{x}=\bar{N}_{y}=0$. The assignment $N_{y}=1, p=\tilde{p}=0$ corresponds to the KK (Cartan field) mode $A_{\mu}^{3}$, while for $N_{y}=0, p=\tilde{p}= \pm 1$ (namely, $k= \pm \frac{2}{\sqrt{\alpha^{\prime}}}, \bar{k}=0$ ), we get the charged vectors $A_{\mu}^{ \pm}$, respectively. The corresponding vertex operators are defined as

$$
V^{i}(z, \bar{z})=i \frac{g_{c}^{\prime}}{\alpha^{\prime 1 / 2}} \epsilon_{\mu}^{3}: J^{i}(z) \bar{\partial} X^{\mu} e^{i K \cdot X}:
$$

where $i= \pm, 3$ and $z=\exp (-i \sigma+\tau)$.

2. The vector states generating $\mathrm{SU}(2)_{R}$ have $N_{x}=1, \bar{N}_{x}=N_{y}=0$. Setting $\bar{N}_{y}=$ $1, p=\tilde{p}=0$ we get $\bar{A}_{\mu}^{3}$, and for $\bar{N}_{y}=0, p=-\tilde{p}= \pm 1$ (i.e. $k=0, \bar{k}= \pm \frac{2}{\sqrt{\alpha^{\prime}}}$ ) we obtain $A_{\mu}^{ \pm}$. The vertex operators associated to these states are given by

$$
\bar{V}^{i}(z, \bar{z})=i \frac{g_{c}^{\prime}}{\alpha^{\prime 1 / 2}} \bar{\epsilon}_{\mu}^{i}: \partial X^{\mu} \bar{J}^{i}(\bar{z}) e^{i K \cdot X}:
$$

3. The nine scalars are obtained after setting $N_{x}=\bar{N}_{x}=0$. They are listed below.

(a) $N_{y}=1, \bar{N}_{y}=1, p=\tilde{p}=0 \quad: M_{33}$

(b) $N_{y}=1, \bar{N}_{y}=0, p=-\tilde{p}= \pm 1\left(k=0, \bar{k}= \pm \frac{2}{\sqrt{\alpha^{\prime}}}\right): \quad M_{3 \pm}$

(c) $N_{y}=0, \bar{N}_{y}=1, p=\tilde{p}= \pm 1\left(k= \pm \frac{2}{\sqrt{\alpha^{\prime}}}, \bar{k}=0\right) \quad: \quad M_{ \pm 3}$

(d) $\bar{N}_{y}=N_{y}=0, p= \pm 2, \tilde{p}=0\left(k=\bar{k}= \pm \frac{2}{\sqrt{\alpha^{\prime}}}\right) \quad: \quad M_{ \pm \pm}$

(e) $\bar{N}_{y}=N_{y}=0, p=0, \tilde{p}= \pm 2\left(k=-\bar{k}= \pm \frac{2}{\sqrt{\alpha^{\prime}}}\right) \quad: \quad M_{ \pm \mp}$

The corresponding vertex operators read

$$
V^{i j}(z, \bar{z})=g_{c}^{\prime} \phi_{i j}: J^{i}(z) \bar{J}^{j}(\bar{z}) e^{i K \cdot X}:
$$

\footnotetext{
${ }^{2}$ We denote by $\epsilon=\epsilon(K)$ with adequate indices the polarizations in momentum space and present its corresponding field. For instance $\epsilon_{\mu}^{3}$ correspond to polarizations of the vector field $A_{\mu}^{3}, \epsilon^{i j}$ are the Fourier components of the scalar $M_{i j}$, etc.
} 
In these expressions, $\mu=0, \ldots, d-1$ is an index along the non-compactified (external) dimensions and $J^{i}$ denote the $\mathrm{SU}(2)_{L}$ currents. $^{3}$ In the standard basis the currents are explicitly given by

$$
\begin{aligned}
& J^{1}(z)=: \cos \left(2 \alpha^{\prime-1 / 2} y(z)\right): \\
& J^{2}(z)=: \sin \left(2 \alpha^{\prime-1 / 2} y(z)\right): \\
& J^{3}(z)=\frac{i}{\sqrt{\alpha^{\prime}}} \partial_{z} y(z) .
\end{aligned}
$$

They satisfy the OPE

$$
J^{i}(z) J^{j}(0) \sim \frac{\kappa^{i j}}{z^{2}}+i \frac{f^{i j} k}{z} J^{k}(0)+\ldots
$$

the Cartan metric being given by $\kappa^{i j}=\frac{1}{2} \delta^{i j}$ and the structure constants being

$$
f^{i j k}=\kappa^{k l} f^{i j}=\frac{1}{2} \epsilon^{i j k} .
$$

The Cartan-Weyl basis is obtained after setting $J^{ \pm}(z)=J^{1}(z) \pm i J^{2}(z)$, namely,

$$
J^{ \pm}(z)=: \exp \left( \pm 2 \alpha^{\prime-1 / 2} y(z)\right): \text {. }
$$

Currents in this basis satisfy the same OPE (2.14) with $\kappa^{++}=\kappa^{--}=0, \kappa^{+-}=1$ and $f^{+-3}=i$.

The factor

$$
g_{c}^{\prime}=g_{c}(2 \pi R)^{-1 / 2}
$$

is the standard $d$-dimensional closed string coupling written in terms of the original $D=$ $d+1$ dimensional coupling $g_{c}$, with the factor $(2 \pi R)^{-1 / 2}$ coming from the normalization of the zero mode wave function.

Concerning the vector polarizations, recall that they must satisfy the gauge condition

$$
K \cdot \epsilon^{i}(K)=K \cdot \bar{\epsilon}^{i}(K)=0
$$

in order for the vertex operators to have the correct conformal weight $(1,1)$.

The names of the modes indicate their transformation properties under the $\mathrm{SU}(2)_{L} \times$ $\mathrm{SU}(2)_{R}$ enhanced gauge symmetry: the $\mathrm{KK}$ vector $A_{\mu}^{3}$ corresponding to the fluctuations of $g_{\mu y}+B_{\mu y}$ combines with $A_{\mu}^{ \pm}$containing discrete momentum and winding modes and they all enhance the $\mathrm{U}(1)_{L}$ to $\mathrm{SU}(2)_{L}$, and similarly $\bar{A}_{\mu}^{3}=g_{\mu y}-B_{\mu y}$ combines with $\bar{A}_{\mu}^{ \pm}$to enhance the $\mathrm{U}(1)_{R}$ to $\mathrm{SU}(2)_{R}$. The scalar $M_{33}$ is related to the fluctuations of the metric $g_{y y}$ which, as we will discuss later, measures the deviation from the self-dual radius. It is massless also away from the self-dual radius, and its (unfixed) vacuum expectation value breaks the $\mathrm{SU}(2)_{L} \times \mathrm{SU}(2)_{R}$ to $\mathrm{U}(1)_{L} \times \mathrm{U}(1)_{R}$ through a stringy Higgs mechanism, as we will see clearly from the effective action. We must also consider the well known vertex operator creating states in the common gravity sector

$$
V_{G}(z, \bar{z})=-\frac{g_{c}^{\prime}}{\alpha^{\prime}} \epsilon_{\mu \nu}^{G} \partial X^{\mu} \bar{\partial} X^{\nu} e^{i K \cdot X},
$$

\footnotetext{
${ }^{3}$ The $\mathrm{SU}(2)_{R}$ current algebra is obtained just by replacing $J^{i}(z) \rightarrow \bar{J}^{i}(\bar{z}), y(z) \rightarrow \bar{y}(\bar{z})$ and $\epsilon_{i j k} \rightarrow \bar{\epsilon}_{i j k}$.
} 
where the label $G=g, B, \varphi$ stands for graviton, antisymmetric tensor and dilaton, respectively, and the polarizations satisfy the usual gauge constraints.

As a closing remark recall that the level matching condition (2.7) can be recast as an eigenvalue equation for vertex operators as

$$
\partial_{Y} \partial_{\tilde{Y}} V_{(p, \tilde{p})}(Y, \tilde{Y})=-p \cdot \tilde{p} V_{(p, \tilde{p})}(Y, \tilde{Y})=(N-\bar{N}) V_{(p, \tilde{p})}(Y, \tilde{Y}) .
$$

\subsection{Effective action at the self-dual radius and Higgs mechanism}

Computation of the three-point amplitudes allows to identify the kinetic and (three field) interaction terms in an effective low energy field theory limit. These amplitudes (given in the appendix) can be reproduced, at the self-radius and up to two derivatives, by the following terms in the action

$$
\begin{aligned}
S=\int d^{d} x & \sqrt{g} e^{-2 \varphi}\left[\frac{1}{2 \kappa_{d}^{2}}\left(\mathcal{R}+4(\partial \varphi)^{2}-\frac{1}{12} H^{2}\right)-\frac{1}{8} F_{\mu \nu}^{i} F^{i \mu \nu}\right. \\
& \left.-\frac{1}{8} \bar{F}_{\mu \nu}^{i} \bar{F}^{i \mu \nu}-\frac{1}{2} g_{d} \sqrt{\alpha^{\prime}} M_{i j} F_{\mu \nu}^{i} \bar{F}^{j \mu \nu}-D_{\mu} M_{i j} D_{\nu} M_{i j} g^{\mu \nu}+\frac{16 g_{d}}{\sqrt{\alpha^{\prime}}} \operatorname{det} M\right],
\end{aligned}
$$

where

$$
\begin{aligned}
H & =d B+A^{i} \wedge F^{i}-\bar{A}^{i} \wedge \bar{F}^{i}, \\
F^{i} & =d A^{i}+g_{d} \epsilon^{i j k} A^{j} \wedge A^{k}, \quad \bar{F}^{i}=d \bar{A}^{i}+g_{d} \bar{\epsilon}^{i j k} \bar{A}^{j} \wedge \bar{A}^{k}, \\
D_{\mu} M_{i j} & =\partial_{\mu} M_{i j}+g_{d} \epsilon^{i k l} A_{\mu}^{k} M_{l j}+g_{d} \bar{\epsilon}^{j k l} \bar{A}_{\mu}^{k} M_{i l}
\end{aligned}
$$

and $g_{d}=\kappa_{d} \sqrt{\frac{2}{\alpha^{\prime}}}$ is the effective gauge coupling constant that we will deduce in the next section. ${ }^{4}$

As expected, this action reproduces an $\mathrm{SU}(2)_{L} \times \mathrm{SU}(2)_{R}$ gauge field theory with nine scalars and coupled to the gravity sector. Details of this kind of computation can be extracted from the literature [21-25]. Actually this expression can also be recovered from the effective action away from the self-dual radius by considering the limit $R \rightarrow \tilde{R}$.

From this action, one can see some of the features of the spontaneous breaking of $\mathrm{SU}(2)_{L} \times \mathrm{SU}(2)_{R}$ into $\mathrm{U}(1)_{L} \times \mathrm{U}(1)_{R}$ that happens away from the self-dual radius. An effective built up stringy Higgs mechanism is already encoded in the string theory computation away from the self-dual point as we shall discuss. Interestingly enough, it can be interpreted as triggered by the vacuum expectation value of the scalar $M_{33}$.

Shifting

$$
M_{33}=v+M_{33}^{\prime},
$$

with $\left\langle M_{33}^{\prime}\right\rangle=0$, we see that the $A^{ \pm}, \bar{A}^{ \pm}$vectors acquire a mass while $A^{3}$ and $\bar{A}^{3}$ remain massless. Some of the scalars also acquire a mass. The vector masses come from the terms

$$
\left(D_{\mu} M_{ \pm 3}\right)^{2}=\left(\partial_{\mu} M_{ \pm 3} \pm 2 g_{d} v A_{\mu}^{ \pm} \pm 2 g_{d} A_{\mu}^{ \pm} M_{33} \mp 2 g_{d} A^{3} M_{ \pm 3} \mp g_{d} \bar{A}^{ \pm} M_{ \pm \mp} \pm g_{d} \bar{A}^{\mp} M_{ \pm \pm}\right)^{2}
$$

\footnotetext{
${ }^{4}$ Notice that in mass units, by assigning $\left[A_{\mu}^{i}\right]=1$, then $\left[g_{d}\right]=2-\frac{d}{2}=-\left[M_{i j}\right]+1$.
} 
and similarly for $M_{3 \pm}$ after exchanging left and right. The scalar masses, on the other hand, come from

$$
\begin{aligned}
\operatorname{det} M= & v \frac{1}{4}\left(\left|M_{++}\right|^{2}-\left|M_{+-}\right|^{2}\right)+\frac{1}{4} M_{33}^{\prime}\left(\left|M_{++}\right|^{2}-\left|M_{+-}\right|^{2}\right) \\
& +\frac{1}{4} M_{3+}\left(M_{-3} M_{+-}-M_{--} M_{+3}\right)+\frac{1}{4} M_{3-}\left(M_{+3} M_{-+}-M_{++} M_{-3}\right)
\end{aligned}
$$

where in the first line we have used that $M_{-+}=\left(M_{+-}\right)^{*}, M_{--}=\left(M_{++}\right)^{*}$.

From the Higgs mechanism in the effective action (2.21) one obtains therefore four massive scalars, $M_{ \pm \pm}, M_{ \pm \mp}$. Recall from section 2.1 that these are states that have only winding or discrete momentum, and no oscillation modes along the circle. Note that half of them acquire a negative mass. ${ }^{5}$ This is because we are dealing with the bosonic string, where the ground state is a tachyon. When $R<R_{\text {sd }}$, the excited states which have only winding are not massive enough to compensate for the negative energy of the tachyon. In the heterotic string, such problem does not arise, and all the states have positive mass.

The other four scalars $M_{3 \pm}$ and $M_{ \pm 3}$ are the massless Goldstone bosons which are eaten by the vectors $A^{ \pm}, \bar{A}^{ \pm}$to become massive, with mass $m_{-}$.

In the next section we present the relevant ingredients of the computation when $R \neq \tilde{R}$ and leave some details for the appendix.

\section{Away from the self-dual point}

The study of amplitudes and the effective field theory away from the self-dual radius is enlightening. Several of their expected features have been discussed in the literature (see for instance [22-24]), but as far as we know, an explicit computation is not yet available.

Interestingly enough, even if three-point amplitudes can only lead to three-field vertices in the effective action, we will see that, away from the self-dual radius, some information about higher order contributions can be guessed.

\subsection{Vertex operators and amplitudes for $R \neq \tilde{R}$}

In this section we present the relevant vertex operators and indicate general aspects of the computation of three-point functions. More details can be found in the appendix.

As can be checked from the mass formula (2.6), when moving away from the self-dual radius, the KK states remain massless while the vectors and scalars with non-vanishing winding and/or compact momentum, acquire a mass. The dependence on the radius in the vertex operators is contained in the exponential factor of the internal coordinates. Namely, these vertices contain a factor

$$
: \exp [i k y(z)+i \bar{k} \bar{y}(\bar{z})] e^{i K \cdot X}:
$$

that will generically depend on the left and right internal coordinates since neither $k$ nor $\bar{k}$ is zero for the states with compact momentum and/or winding away from the self-dual radius.

\footnotetext{
${ }^{5}$ Which half are tachyonic depends on the sign of $v$. For $v>0$, which as we will see later corresponds to $R>\tilde{R}$, or in other words $R>\sqrt{\alpha^{\prime}}$, the states $M_{ \pm \pm}$that have momentum are tachyonic, while those with winding, $M_{ \pm \mp}$, have positive mass, as expected.
} 
In particular the $\mathrm{U}(1)_{L} \times \mathrm{U}(1)_{R}$ charges of these states, generated by $J^{3} \oplus \bar{J}^{3}$, will be $(q, \bar{q})=\sqrt{\alpha^{\prime}}\left(\frac{k}{2}, \frac{\bar{k}}{2}\right)$. Notice that under a T-duality transformation exchanging the radius $R$ with the dual radius $\tilde{R}$ and windings with compact momenta

$$
q \leftrightarrow q, \quad \bar{q} \leftrightarrow-\bar{q} .
$$

To be more explicit, let us discuss the case of the vector states with one unit of winding and compact momentum. These become massive away from the self-dual radius with $M_{V^{ \pm}}=$ $m_{-}$, where $m_{-}$is defined in (2.9), and have $k=m_{+}, \bar{k}=m_{-}$. Therefore, the operators

$$
V^{ \pm}(z, \bar{z})=i \frac{g_{c}^{\prime}}{\alpha^{\prime 1 / 2}} \epsilon_{\mu}^{ \pm}: \bar{\partial} X^{\mu} e^{i K \cdot X} \exp \left[ \pm i m_{+} y(z)\right] \exp \left[ \pm i m_{-} \bar{y}(\bar{z})\right]:
$$

reduce to the $\mathrm{SU}(2)_{L}$ massless vector vertices (2.10) for $R=\tilde{R}$ and then might seem appropriate to create the gauge bosons $A_{\mu}^{ \pm}$.

However, although they have the correct conformal weight $(h, \bar{h})=(1,1)$, a cubic anomaly proportional to $K \cdot \epsilon^{ \pm}$appears in the OPE with the anti-holomorphic energymomentum tensor, and the transverse polarization conditions (2.18) canceling the anomalies in the massless case are not correct here on physical grounds since these vectors are massive.

To build the appropriate vertex operators, it is instructive to consider the scalars that should provide the longitudinal components of the polarization, denoted $V^{ \pm 3}$. Their vertex operators are

$$
V^{ \pm 3}(z, \bar{z})=i \frac{g_{c}^{\prime}}{\alpha^{1 / 2}} \phi_{ \pm 3} \bar{\partial} \bar{y}(\bar{z}) e^{ \pm i m_{+} y} e^{ \pm i m_{-} \bar{y}} e^{i K \cdot X}
$$

These states have the same mass, $M_{V^{ \pm 3}}=m_{-}$, and an anomalous cubic contribution proportional to $\phi_{ \pm 3}\left( \pm m_{-}\right)$that cannot be set to zero away from the self-dual radius. Intriguingly, this anomaly can be canceled against that of the massive vectors! Indeed, considering the combinations

$$
V^{\prime \pm}=V^{ \pm}-\xi V^{ \pm 3}
$$

where $\xi$ is some coefficient, the anomalies cancel provided

$$
K \cdot \epsilon^{ \pm} \mp \xi m_{-} \phi_{ \pm 3}=0,
$$

which reduces to the transverse polarization condition (2.18) for the massless states.

In terms of fields, these equations would read

$$
\partial_{\mu} A^{ \pm \mu} \pm i \xi m_{-} M_{ \pm 3}=0 .
$$

Interestingly enough, this is just the $R_{\xi}$ t'Hooft gauge condition in gauge theories

$$
f^{i}=\partial \cdot A^{i}-i g_{d} \xi \mathbf{T}^{i} v \phi=0,
$$

where $\mathbf{T}^{i}$ are generators, $\phi$ scalars and $v$ the vev of the scalar field. Namely, in our case for $\mathrm{SU}(2) \times \mathrm{SU}(2):\left(\mathbf{T}^{i}\right) j k=-i \epsilon^{i j k}$, and $\phi \equiv M_{i j}$, with non-vanishing vev $v_{33}$ such that

$$
f^{i}=\partial_{\mu} A^{i \mu}-g_{d} \xi \epsilon^{i j k} v^{k l} M_{j l}
$$


and defining $g_{d} v^{33}=g_{d} v=-m_{-}$, we obtain (3.7). We see here an indication that the generation of masses can be given an interpretation in terms of a Higgs mechanism in the effective theory.

We learn by this that the physical massive vector boson vertices are actually $V^{\prime \pm}$, and the scalars $M_{ \pm 3}$ should disappear from the spectrum. Notice that the fields associated to $V^{\prime \pm}$ have well defined charges $(q, \bar{q})= \pm \frac{\sqrt{\alpha^{\prime}}}{2}\left(m_{+}, m_{-}\right)$. Moreover, since for the massive vectors $K^{2}=-m_{-}^{2}$, the gauge condition (3.6) can be rewritten as

$$
0=K \cdot \epsilon^{ \pm} \mp \xi m_{-} \phi_{ \pm 3}=K \cdot\left(\epsilon^{ \pm} \pm K \xi \frac{1}{m_{-}} \phi_{ \pm 3}\right) .
$$

This implies that

$$
\epsilon_{\mu}^{\prime \pm}=\epsilon_{\mu}^{ \pm} \pm \xi K_{\mu} \frac{1}{m_{-}} \phi_{ \pm 3}
$$

appears as an effective polarization. In terms of fields this polarization leads to a massive vector of the form

$$
A_{\mu}^{\prime \pm}=A_{\mu}^{ \pm} \pm \frac{i}{m_{-}} \xi \partial_{\mu} M_{ \pm 3} \equiv A_{\mu}^{ \pm} \mp \frac{i}{q v} \xi \partial_{\mu} M_{ \pm 3}
$$

where the vev $v^{2}=m_{-}^{2} \neq 0$ and $q= \pm$ is the $\mathrm{U}(1)$ charge of $A_{\mu}$. This is the usual massive vector field incorporating the Goldstone boson that provides the longitudinal polarization.

A parallel situation holds for the vertex operators creating the $\mathrm{SU}(2)_{R}$ vectors $\bar{V}^{ \pm}(z, \bar{z})$, where we need to define the massive vector field

$$
\bar{V}^{\prime \pm}=\bar{V}^{ \pm}-\bar{\xi} V^{3 \pm}
$$

and gauge condition

$$
K \cdot \bar{\epsilon}^{ \pm} \mp \bar{\xi} m_{-} \phi_{3 \pm}=0 .
$$

The corresponding expressions for the vector fields and effective polarizations are obtained just by exchanging barred and unbarred quantities.

The two scalars $M_{ \pm \mp}$ with $M^{2}=\frac{4}{\tilde{R}^{2}}-\frac{4}{\alpha^{\prime}}=-\frac{4}{\widetilde{R}} m_{-}$and $(q, \bar{q})= \pm \frac{\sqrt{\alpha^{\prime}}}{\tilde{R}}(1,-1)$ have vertex operators

$$
V^{ \pm \mp}(z, \bar{z})=g_{c}^{\prime} \frac{1}{4} \phi_{ \pm \mp}: e^{ \pm \frac{2 i}{\bar{R}} y(z)} e^{\mp \frac{2 i}{R} \bar{y}(\bar{z})} e^{i K \cdot X(z, \bar{z})}:
$$

while the vertices for the scalars $M_{ \pm \pm}$with $M^{2}=\frac{4}{R^{2}}-\frac{4}{\alpha^{\prime}}=\frac{4}{R} m_{-}$and $(q, \bar{q})= \pm \frac{\sqrt{\alpha^{\prime}}}{R}(1,1)$ are

$$
V^{ \pm \pm}(z, \bar{z})=g_{c}^{\prime} \frac{1}{4} \phi_{ \pm \mp}: e^{ \pm \frac{2 i}{R} y(z)} e^{ \pm \frac{2 i}{R} \bar{y}(\bar{z})} e^{i K \cdot X(z, \bar{z})}:
$$

We see that away from the self-dual radius, two states become massive and two tachyonic depending on $R>\tilde{R}$ or $R<\tilde{R}$. This is in agreement with what we get from the Higgs mechanism based on the effective action at the self-dual radius, as discussed in the previous section. We will comment more on this in section 3.3.

Finally, $M_{33}$ remains massless and thus its vertex operator is (2.12). 
It is useful to note how fields transform under T-duality. For instance,

$$
\begin{aligned}
M_{ \pm \pm} & \leftrightarrow M_{ \pm \mp} & M_{33} & \leftrightarrow-M_{33} \\
A^{3} & \leftrightarrow A^{3} & \bar{A}^{3} & \leftrightarrow-\bar{A}^{3} \\
A^{ \pm} & \leftrightarrow A^{ \pm} & \bar{A}^{ \pm} & \leftrightarrow \bar{A}^{\mp} .
\end{aligned}
$$

On the eaten scalars $M_{ \pm 3}, M_{3 \pm}$, on the other hand, it acts as

$$
M_{ \pm 3} \leftrightarrow-M_{ \pm 3} \quad M_{3 \pm} \leftrightarrow M_{3 \mp} .
$$

Both (3.17) and (3.18) can be recast as the right multiplication by

$$
D=\left(\begin{array}{ccc}
0 & 1 & 0 \\
1 & 0 & 0 \\
0 & 0 & -1
\end{array}\right)
$$

on the matrix $M_{i j}, i, j= \pm, 3$, for the scalars and on the row vector $\bar{A}^{i}$ for the bosons. If a trivial left multiplication is also assumed on $M_{i j}$, which transform as $(3,3)$ under $\mathrm{SU}(2)_{L} \times \mathrm{SU}(2)_{R}$, T-duality can be recognized as a $\mathbb{Z}_{2}$ symmetry included in the enhanced gauge group. Indeed, the idempotent matrix $D$ belongs to the adjoint representation of $\mathrm{SU}(2)$, corresponding to a rotation by $\pi$ around the 2 -axis. As it was already pointed out in [24], this fact shows that T-duality is a symmetry not only of the perturbation theory but of the exact string theory.

We expect that amplitudes involving the states that are massive away from the self-dual radius are well defined and that the physical massive vectors (3.5) and (3.13) give sensible results independently of the value of $\xi, \bar{\xi}$, provided the gauge fixing conditions (3.6), (3.14) are used. In what follows we show that this is indeed the case.

\subsection{Effective action from string theory at $R \neq \tilde{R}$}

In this section we sketch the computation of the string theory three-point functions on the sphere and obtain the classical effective action at the two derivative level for the closed bosonic string compactified on a circle of radius $R$ close to the self-dual radius (2.5). Some details are given in the appendix.

Only states that are massless at the self-dual radius are considered. As we discussed in the previous section, some of these acquire a mass of order $m_{-}$away from the self-dual radius and a Higgs mechanism interpretation can be invoked in the effective field theory, as we will show in detail in section 3.3. We introduce a small parameter $\epsilon$, which measures deviations away from the self-dual radius, in the form ${ }^{6}$

$$
R=\sqrt{\alpha^{\prime}} \exp (\epsilon)=\sqrt{\alpha^{\prime}}\left(1+\epsilon+\mathcal{O}\left(\epsilon^{2}\right)\right) .
$$

\footnotetext{
${ }^{6}$ The reason for this choice of parametrization will become clear later, when we relate this to the vev of $M_{33}$.
} 
Therefore, the parameters introduced in (2.9) read

$$
\begin{aligned}
& m_{-}=\frac{1}{\alpha^{\prime}}(\tilde{R}-R)=-\frac{2}{\sqrt{\alpha^{\prime}}} \sinh (\epsilon) \approx-\frac{2}{\sqrt{\alpha^{\prime}}} \epsilon, \\
& m_{+}=\frac{1}{\alpha^{\prime}}(\tilde{R}+R)=\frac{2}{\sqrt{\alpha^{\prime}}} \cosh (\epsilon) \approx \frac{2}{\sqrt{\alpha^{\prime}}} .
\end{aligned}
$$

In order for the effective action description to be valid, the mass scales $E$ involved should be such that $E \sim\left|m_{-}\right|=|\epsilon| / \sqrt{\alpha^{\prime}} \ll 1 / \sqrt{\alpha^{\prime}}$, and other massive states should be neglected. Then, we compute all possible three-point amplitudes involving the gravity sector massless states, the massless gauge bosons $A^{3}, \bar{A}^{3}$, the massless scalar $M_{33}$, the massive scalars $M_{ \pm \pm}, M_{ \pm \mp}$ and the massive vectors $A^{\prime \pm}, \bar{A}^{\prime \pm}$.

We showed that the well defined massive vectors have vertex operators of the form $V^{\prime \pm}=V^{ \pm}-\xi V_{ \pm 3}$, where $V_{ \pm 3}$ is associated to the Goldstone boson $M_{ \pm 3}$. Therefore, amplitude computations would require partial evaluations involving $V^{ \pm}$and $V_{ \pm 3}$. For instance, the computation of the three-point amplitude $\left\langle V^{\prime \pm}\left(z_{1}\right) V^{\prime \mp}\left(z_{2}\right) V_{3}\left(z_{3}\right)\right\rangle$ splits into four components. The partial amplitudes will be ill-defined since the component fields are anomalous and generically the conformal volume will not factorize. This manifests as a mismatching of powers of $z_{i j}=z_{i}-z_{j}$ and $\bar{z}_{i j}=\bar{z}_{i}-\bar{z}_{j}$ that do not reconstruct the conformal volume factor $\left|z_{12} z_{23} z_{13}\right|^{2}$ that must factorize. It is after summing up partial results and using the gauge condition (3.6) that a sensible amplitude is obtained.

It is possible to check that the amplitudes involving massive vectors $V^{\prime \pm}(\epsilon)$ with other vertices produce the same results as those amplitudes calculated with the massless operator $V^{ \pm}\left(\epsilon^{\prime}\right)$ but using the effective polarization $\epsilon^{\prime}$ introduced in (3.11). From the practical point of view this observation leads to an important simplification. For instance only one correlator, instead of four, needs to be computed in the amplitude involving three vectors. The explicit computations are straightforward and follow the well-known steps given in textbooks. We summarize these steps below and show a couple of relevant examples.

- Write down the three-point amplitude and perform all contractions with the propagators

$$
\begin{aligned}
\left\langle X^{\mu}(z, \bar{z}) X^{\nu}(w, \bar{w})\right\rangle & =-\frac{\alpha^{\prime}}{2} \eta^{\mu \nu} \ln |z-w|^{2}, \\
\langle y(z) y(w)\rangle & =-\frac{\alpha^{\prime}}{2} \ln (z-w), \\
\langle\bar{y}(\bar{z}) \bar{y}(\bar{w})\rangle & =-\frac{\alpha^{\prime}}{2} \ln (\bar{z}-\bar{w})
\end{aligned}
$$

- Cancel out the volume of the conformal group.

- Include the normalization factor $C_{S^{2}}=\frac{8 \pi}{\alpha^{\prime} g_{c}^{\prime 2}}$.

In order to find the effective field theory, we must compare the results of the amplitudes with terms involving three-field interactions in the field theory Lagrangian written in momentum space. Namely, a field will appear in terms of its corresponding polarization and momenta as derivatives. Namely,

$$
\epsilon_{\mu}(K) \leftrightarrow A_{\mu}(x)
$$




$$
\begin{aligned}
\phi_{ \pm \pm}(K) & \leftrightarrow M_{ \pm \pm}(x) \\
\varepsilon_{\mu \nu}^{g}(K) & \leftrightarrow h_{\mu \nu}(x) \\
i K_{\mu} & \leftrightarrow \partial_{\mu}
\end{aligned}
$$

where we must consider fluctuations around the flat metric $g_{\mu \nu}(x) \simeq \eta_{\mu \nu}+2 \kappa_{d} h_{\mu \nu}(x)$.

For instance, in order to obtain the kinetic terms for the scalar fields $M_{ \pm \pm}$, we first compute

$$
\left\langle V^{ \pm \pm} V^{\mp \mp} V_{G}\right\rangle=-\frac{1}{8} g_{c}^{\prime} \pi \delta_{k} \phi_{ \pm, \pm} \phi_{\mp \mp} \varepsilon_{\mu \nu}^{G} k_{1}^{\mu} k_{2}^{\nu},
$$

where $\delta_{k}=(2 \pi)^{d} \delta^{d}\left(\sum_{i=1}^{3} k_{i}\right)$, leading to the term

$$
\frac{1}{8} g_{c}^{\prime} \pi \partial_{\mu} M_{ \pm \pm} \partial_{\nu} M_{\mp \mp} h^{\mu \nu} .
$$

This reproduces the coupling with metric fluctuations $\frac{1}{2 \kappa_{d}^{2}} \partial_{\mu} M_{ \pm \pm} \partial_{\nu} M_{\mp \mp} h^{\mu \nu}$ if

$$
\frac{1}{8} g_{c}^{\prime} \pi=2 \kappa_{d} C
$$

where $\mathrm{C}$ is a constant coming from de LSZ Theorem. Moreover, by computing

$$
\begin{aligned}
\left\langle V^{ \pm \pm}\left(z_{1}\right) V^{\mp \mp}\left(z_{2}\right) V^{3}\left(z_{3}\right)\right\rangle & =\frac{1}{8} g_{c}^{\prime} \pi \phi_{ \pm \pm} \phi_{\mp \mp}\left(\epsilon^{3} \cdot K_{2}-\epsilon^{3} \cdot K_{1}\right) \frac{1}{R} \delta_{k} \\
\left\langle V^{ \pm \pm}\left(z_{1}\right) V^{\mp \mp}\left(z_{2}\right) \bar{V}^{3}\left(z_{3}\right)\right\rangle & =\frac{1}{8} g_{c}^{\prime} \pi \phi_{ \pm \pm} \phi_{\mp \mp}\left(\bar{\epsilon}^{3} \cdot K_{2}-\bar{\epsilon}^{3} \cdot K_{1}\right) \frac{1}{R} \delta_{k},
\end{aligned}
$$

we can read off the following terms in the effective action

$$
-i \frac{1}{8} g_{c}^{\prime} \pi\left[\partial_{\mu} M_{++}\left(\frac{1}{R} A_{\mu}^{3}+\frac{1}{R} \bar{A}_{\mu}^{3}\right) M_{--}-M_{++}\left(\frac{1}{R} A_{\mu}^{3}+\frac{1}{R} \bar{A}_{\mu}^{3}\right) \partial_{\mu} M_{--}\right]
$$

and therefore, combining this with (3.24), we can write the kinetic term in the effective action

$$
-\frac{1}{2\left(2 \kappa_{d}^{2}\right)} D_{\mu} M_{ \pm \pm} D_{\nu} M_{\mp \mp} g^{\mu \nu}
$$

with

$$
D_{\mu}=\partial_{\mu}-i g q A_{\mu}^{3}-i g \bar{q} \bar{A}_{\mu}^{3}
$$

where $(q, \bar{q})=\frac{\sqrt{\alpha^{\prime}}}{R}(1,1)$ are the corresponding $\mathrm{U}(1)_{L} \times \mathrm{U}(1)_{R}$ charges.

In order to have a standard normalization we redefine the scalar and vector fields to absorb the factor $\frac{1}{2 \kappa_{d}^{2}}$.

In doing so we notice that the amplitude results coincide with the ones obtained from the effective action if the gauge and gravitational couplings are related as

$$
g_{d}=\frac{\kappa_{d} \sqrt{2}}{\sqrt{\alpha^{\prime}}}
$$

Proceeding in the same manner for the other terms, we can finally write the low energy effective action, up to cubic interactions. Terms containing four fields can be completed by invoking gauge invariance. We find,

$$
S_{R \neq \tilde{R}}=\int d^{d} x \sqrt{g} e^{-2 \varphi}\left[\frac{1}{2 \kappa_{d}^{2}}\left(\mathcal{R}+4\left(\partial_{\mu} \varphi\right)^{2}-\frac{1}{12} H_{\mu \nu \rho} H^{\mu \nu \rho}\right)\right.
$$




$$
\begin{aligned}
& -\frac{1}{8} F_{\mu \nu}^{3} F^{\mu \nu 3}-\frac{1}{8} \bar{F}_{\mu \nu}^{3} \bar{F}^{\mu \nu 3} \\
& -\frac{1}{8}{F^{\prime}}_{\mu \nu}^{+} F^{\prime \mu \nu-}-\frac{1}{4} m_{-}^{2} A_{\mu}^{\prime} A_{\nu}^{\prime} g^{\mu \nu}-\frac{1}{8} \bar{F}_{\mu \nu}^{\prime+} \bar{F}^{\prime \mu \nu-}-\frac{1}{4} m_{-}^{2} \bar{A}_{\mu}^{\prime} \bar{A}_{\nu}^{\prime} g^{\mu \nu} \\
& -\partial_{\mu} M_{33} \partial^{\mu} M_{33}-\frac{1}{2} D_{\mu} M_{ \pm \pm} D^{\mu} M_{\mp \mp}-\frac{1}{2} D_{\mu} M_{ \pm \mp} D^{\mu} M_{\mp \pm} \\
& +i g_{d} \frac{\sqrt{\alpha^{\prime}} m_{+}}{2} A^{\prime}+\mu A^{\prime-\nu} F_{\mu \nu}^{3}+i g_{d} \frac{\sqrt{\alpha^{\prime}} m_{-}}{2} A^{\prime}+\mu A^{\prime}-\nu \bar{F}_{\mu \nu}^{3} \\
& +i g_{d} \frac{\sqrt{\alpha^{\prime}} m_{+}}{2} \bar{A}^{\prime}+\mu \bar{A}^{\prime}-\nu \bar{F}_{\mu \nu}^{3}+i g_{d} \frac{\sqrt{\alpha^{\prime}} m_{-}}{2} \bar{A}^{\prime}+\mu \bar{A}^{\prime}-\nu F_{\mu \nu}^{3} \\
& +g_{d} \frac{m_{+} \sqrt{\alpha^{\prime}}}{2} A^{\prime+\mu} A_{\mu}^{\prime-} M_{33} m_{-}+g_{d} \frac{m_{+} \sqrt{\alpha^{\prime}}}{2} \bar{A}^{\prime+\mu} \bar{A}_{\mu}^{\prime-} M_{33} m_{-} \\
& -g_{d} \sqrt{\alpha^{\prime}} \frac{1}{8} F_{\mu \nu}^{\prime \pm} \bar{F}^{\prime \pm \mu \nu} M_{\mp \mp}-g_{d} \sqrt{\alpha^{\prime}} \frac{1}{8} F_{\mu \nu}^{\prime \pm} \bar{F}^{\prime} \mp \mu \nu M_{\mp \pm}-g_{d} \sqrt{\alpha^{\prime}} \frac{1}{2} F_{\mu \nu}^{3} \bar{F}^{3 \mu \nu} M_{33} \\
& -\frac{4 g_{d}}{\sqrt{\alpha^{\prime}}} M_{+-} M_{-+} M_{33}\left(\frac{\sqrt{\alpha^{\prime}}}{\tilde{R}}\right)^{2}+\frac{4 g_{d}}{\sqrt{\alpha^{\prime}}} M_{++} M_{--} M_{33}\left(\frac{\sqrt{\alpha^{\prime}}}{R}\right)^{2} \\
& \left.+\frac{4 m_{-}}{\tilde{R}} \frac{1}{2} M_{+-} M_{-+}-\frac{4 m_{-}}{R} \frac{1}{2} M_{++} M_{--}\right]
\end{aligned}
$$

with $^{7}$

$$
\begin{gathered}
F_{\mu \nu}^{\prime \pm}=2 \partial_{[\mu} A_{\nu]}^{\prime \pm} \mp i g_{d} \frac{\sqrt{\alpha^{\prime}} m_{+}}{2} 2 A_{[\mu}^{3} A_{\nu]}^{\prime \pm} \mp i g_{d} \frac{\sqrt{\alpha^{\prime}} m_{-}}{2} 2 \bar{A}_{[\mu}^{3} A_{\nu]}^{\prime \pm} \\
\bar{F}_{\mu \nu}^{\prime \pm}=2 \partial_{[\mu} \bar{A}_{\nu]}^{\prime \pm} \mp i g_{d} \frac{\sqrt{\alpha^{\prime}} m_{+}}{2} 2 \bar{A}_{[\mu}^{3} \bar{A}_{\nu]}^{\prime \pm} \mp i g_{d} \frac{\sqrt{\alpha^{\prime}} m_{-}}{2} 2 A_{[\mu}^{3} \bar{A}_{\nu]}^{\prime \pm} \\
F_{\mu \nu}^{3}=2 \partial_{[\mu} A_{\nu]}^{3} \\
D_{\mu} M_{ \pm \pm}=\left[\partial_{\mu}+i( \pm) g_{d} \frac{\sqrt{\alpha^{\prime}}}{R} A_{\mu}^{3}+i( \pm) g_{d} \frac{\sqrt{\alpha^{\prime}}}{R} \bar{A}_{\mu}^{3}\right] M_{ \pm \pm} \\
D_{\mu} M_{ \pm \mp}=\left[\partial_{\mu}+i( \pm) g_{d} \frac{\sqrt{\alpha^{\prime}}}{\tilde{R}} A_{\mu}^{3}-i( \pm) g_{d} \frac{\sqrt{\alpha^{\prime}}}{\tilde{R}} \bar{A}_{\mu}^{3}\right] M_{ \pm \mp}
\end{gathered}
$$

We have isolated the factor $\frac{\sqrt{\alpha^{\prime}} m_{+}}{2} \rightarrow 1$ at the self-dual radius.

Let us point out some expected, but nevertheless interesting, features of this action. For instance, we see that covariant derivatives correctly appear in terms of the charges of the corresponding fields: $\left(q_{ \pm}, \bar{q}_{ \pm}\right)= \pm \frac{\sqrt{\alpha^{\prime}}}{2}\left(m_{+}, m_{-}\right)$for massive vector bosons $A_{\nu}^{\prime \pm}$, or $\left(q_{ \pm \pm}, \bar{q}_{ \pm \pm}\right)=\frac{\sqrt{\alpha^{\prime}}}{R}(1,1)$ for massive scalars $M_{ \pm \pm}$, etc. Moreover, by keeping track of the original windings and compact momenta it can be checked that the action is invariant under T-duality. For example, by using the transformation properties (3.17) and $R \leftrightarrow \tilde{R}$ we see that charged scalar terms in the action do exchange under T-duality.

It is worth noticing that, in all expressions above, only the combinations

$$
g_{d} \frac{\sqrt{\alpha^{\prime}} m_{+}}{2} A_{\mu}^{3}+g_{d} \frac{\sqrt{\alpha^{\prime}} m_{-}}{2} \bar{A}_{\nu}^{3}=\frac{g_{d} \sqrt{\alpha^{\prime}}}{R} \frac{\left(A_{\mu}^{3}+\bar{A}_{\mu}^{3}\right)}{2}+\frac{g_{d} \sqrt{\alpha^{\prime}}}{\tilde{R}} \frac{\left(A_{\mu}^{3}-\bar{A}_{\mu}^{3}\right)}{2}
$$

\footnotetext{
${ }^{7}$ We define the antisymmetrizer with a $\frac{1}{2}$ factor.
} 
appear. This indicates that, away from the self-dual point, it is sensible to define the fields

$$
V_{\mu}=\frac{1}{2}\left(A_{\mu}^{3}+\bar{A}_{\mu}^{3}\right), \quad B_{\mu}=\frac{1}{2}\left(A_{\mu}^{3}-\bar{A}_{\mu}^{3}\right)
$$

which are the KK reductions of the metric and antisymmetric field.

Interestingly enough, we see that the dependence on the radii can be absorbed in two corresponding gauge couplings

$$
g_{v}=g_{d} \frac{\sqrt{\alpha^{\prime}}}{R} \quad g_{b}=g_{d} \frac{\sqrt{\alpha^{\prime}}}{\tilde{R}} .
$$

In doing so the coupling charges become integers.

\subsection{Interpretation as a Higgsing}

In the string amplitudes there appear terms that have an explicit $R, \tilde{R}$ dependence, and there are also contributions proportional to $m_{-}$that vanish at the self-dual radius. These are a manifestation of a built-in Higgs mechanism. In fact, as sketched at the end of the preceding section, it is instructive to try to interpret these different terms that are generated when slightly sliding away from $R_{s d}$, as coming from giving a vev to the field $M_{33}$ as in (2.23), related to the fluctuations of the radius around the self-dual point.

Consider, as an example, the gauge kinetic terms in (3.31)

$$
-\frac{1}{8} F_{\mu \nu}^{3} F^{\mu \nu 3}-\frac{1}{8} \bar{F}_{\mu \nu}^{3} \bar{F}^{\mu \nu 3}-\frac{1}{2} F_{\mu \nu}^{3} \bar{F}_{\mu \nu}^{3} g_{d} \sqrt{\alpha^{\prime}} M_{33},
$$

where we notice that left and right massless vectors couple through the scalar $M_{33}$. However, the original $V_{\mu}, B_{\mu}$ vectors are decoupled. Namely, in terms of these vectors this is (after redefining the vector fields as $A \rightarrow \frac{1}{g} A$, as usually done)

$$
-\frac{1}{4 g_{v}^{2}} F_{v \mu \nu} F_{v}^{\mu \nu}-\frac{1}{4 g_{b}^{2}} F_{b \mu \nu} F_{b}^{\mu \nu}-\left(\frac{1}{4 g_{v}^{2}} F_{v \mu \nu} F_{v}^{\mu \nu}-\frac{1}{4 g_{b}^{2}} F_{b \mu \nu} F_{b}^{\mu \nu}\right) 2 g_{d} \sqrt{\alpha^{\prime}} M_{33}
$$

It is interesting to interpret the dependence of the coupling constants through the Higgs mechanism. Namely, starting from the self-dual point where $g_{v}^{2}=g_{b}^{2}=\frac{g_{d}^{2}}{\alpha^{\prime}}$ and performing the shift (2.23), we would have, for instance

$$
\begin{aligned}
& -\frac{\alpha^{\prime}}{4 g_{d}^{2}} F_{v \mu \nu} F_{v}^{\mu \nu}-\frac{\alpha^{\prime}}{4 g_{d}^{2}} F_{v \mu \nu} F_{v}^{\mu \nu} 2 g_{d} \sqrt{\alpha^{\prime}}\left(M_{\prime} 33+v\right)+\ldots \\
& \quad=-\frac{\alpha^{\prime}\left(1+2 g_{d} \sqrt{\alpha^{\prime}} v+\ldots\right)}{4 g_{d}^{2}} F_{v \mu \nu} F_{v}^{\mu \nu}-\frac{\alpha^{\prime}}{4 g_{d}^{2}} F_{v \mu \nu} F_{v}^{\mu \nu} g_{d} \sqrt{\alpha^{\prime}} M_{\prime 33}+\ldots
\end{aligned}
$$

where the dots stand for higher order terms in $M_{33}$. If we identify

$$
\alpha^{\prime}\left(1+2 g_{d} \sqrt{\alpha^{\prime}} v+\ldots\right)=R^{2},
$$

we recover the factor $-\frac{1}{4 g_{v}^{2}}$ in (3.37). Furthermore, we expect the exact relation between $R$ and $v$ to be at all orders

$$
\alpha^{\prime} e^{\sqrt{\alpha^{\prime}} 2 g_{d} v}=R^{2} .
$$


This implies that $v$, the vev of $M_{33}$, measures the deviations away from the self-dual radius, and should therefore be small. Recalling the perturbation parameter $\epsilon$ introduced in (3.20), we have that

$$
\sqrt{\alpha^{\prime}} g_{d} v=\epsilon .
$$

Coming back to (3.37), one would then also expect the coupling between $V_{\mu}$ and $M_{33}$ to be at all orders of the form

$$
-\frac{1}{4 g_{d}^{2}} F_{v \mu \nu} F_{v}^{\mu \nu} e^{\sqrt{\alpha^{\prime}} 2 g_{d} M_{33}}
$$

which is the expected contribution from a KK reduction. Similarly the full coupling $g_{b}$ can be reproduced by assuming a coupling $-\frac{1}{4 g_{d}^{2}} F_{b \mu \nu} F_{b}^{\mu \nu} e^{-\sqrt{\alpha^{\prime}} 2 g_{d} M_{33}}$.

Let us discuss now the scalar potential terms and consider the contribution

$$
4 g_{d} \sqrt{\alpha^{\prime}} M_{++} M_{--} M_{33} \frac{1}{R^{2}}
$$

in (3.31), that becomes $4 g_{d} \frac{1}{\sqrt{\alpha^{\prime}}} M_{++} M_{--} M_{33}$ at the self-dual point. If the higher order contributions in $M_{33}$ were of the form $-2 \frac{1}{\alpha^{\prime}}\left(-1+e^{-\sqrt{\alpha^{\prime}} 2 g_{d} M_{33}}\right) M_{++} M_{--}$, the linear term in $M_{33}$ would reproduce the cubic term at the self-dual point, and furthermore, giving a vev to $M_{33}$ we would obtain the precise $R$-dependence of the three-point contribution away from $R_{s d}$. Moreover, the terms that are independent of $M_{33}$ would lead to $4 \frac{1}{R} m_{-} \frac{M_{++} M_{--}}{2}=$ $m_{++}^{2} \frac{M_{++} M_{--}}{2}$, namely the mass term for the complex scalar $M_{++}$. A similar result comes out for the mass term of $M_{+-}$. Thus we see that, even if the three-point amplitudes can only account for three-field-interaction terms in the effective action, when moving away from the self-dual point, we can infer information about higher order terms. Computation of higher order amplitudes should be performed to verify this.

In order to compare this string effective action with results from DFT, we keep only the first order terms in the $\epsilon$-expansion.

\section{$4 \quad$ DFT and enhanced gauge symmetries}

In this section we show how the effective action with enhanced gauge symmetry derived in the last section can be obtained from Double Field Theory.

We first note that after compactifying on a circle at the self-dual radius, with $d$ uncompactified directions, ${ }^{8}$ the spectrum of the bosonic string has $(d+3)^{2}$ massless states: $d^{2}$ from $g_{\mu \nu}$ and $B_{\mu \nu}, 6 d$ from the vector states in items 1 and 2 of section 2.1 and 9 represented by the scalar states in item 3 . This precisely agrees with the dimension of the coset

$$
\frac{O(d+3, d+3)}{O(d+3) \times O(d+3)}
$$

that counts the number of degrees of freedom in the DFT formulation with symmetry $O(d+3, d+3)$, in other words, the number of degrees of freedom in a metric and a $B$

\footnotetext{
${ }^{8}$ Since we are dealing with bosonic string theory, we should take $d+1=26$. However, a similar reasoning works for the common sector of the heterotic string (i.e. ignoring the gauge fields), where $d+1=10$. In the superstring on the other hand there is no enhancement of symmetries at the self-dual point.
} 
field defined in $d+3$ dimensions. Based on this observation, we will work out a covariant $O(d+3, d+3)$ DFT-like formulation that reproduces the effective action computed in the previous section. As a first step, we construct a manifestly $O(d+1, d+1)$ covariant formulation of the theory in $d+1$ dimensions compactified on a circle. And then we extend it to $O(d+3, d+3)$ to account for the enhanced gauge symmetry at the self-dual radius.

We start recalling some notions of DFT that will be needed throughout the rest of the paper.

\subsection{Some notions on DFT}

Here we briefly review some basic features of GCG and/or DFT. The theory is defined on a generalized tangent bundle which locally is $T M \oplus T^{*} M$ and whose sections, the generalized vectors $V$, are direct sums of vectors $v$ plus one forms $\xi$

$$
V=v+\xi
$$

A generalized frame $E_{A}$ on this bundle is a set of linearly independent generalized vectors that belong to the vector space of representations of the group $G=O(D, D)$. It parametrizes the coset $G / G_{c}$, the quotient being over the maximal compact subgroup of $G$, a Lorentz signature assumed on the $D$-dimensional space-time, i.e. $G_{c}=$ $O(1, D-1) \times O(D-1,1)$. Given a frame $e_{a}$ for the tangent bundle $T M$, there is a canonical way to build the generalized frame through the exponentiated action of the $B$ field, namely, ${ }^{9}$

$$
\left(\begin{array}{c}
E_{a} \\
E^{a}
\end{array}\right)=e^{B}\left(\begin{array}{c}
e_{a} \\
e^{a}
\end{array}\right),
$$

where $e^{a}$ in $T^{*} M$ is dual to $e_{a}$ (i.e. $\left.\iota_{e_{a}} e^{b}=\delta_{a}{ }^{b}\right) .{ }^{10}$ Eq. (4.3) explicitly gives

$$
\begin{aligned}
& E_{a}=e_{a}-\iota_{e_{a}} B, \\
& E^{a}=e^{a} .
\end{aligned}
$$

Upper and lower indices distinguish vectors and forms, respectively.

There exists a natural pairing between generalized vectors, namely

$$
V_{1} \cdot V_{2}=\iota_{v_{1}} \xi_{2}+\iota_{v_{2}} \xi_{1}=\eta\left(V_{1}, V_{2}\right)=V_{1}^{M} \eta_{M N} V_{2}^{N}
$$

where $M, N=0,1, \ldots, 2 D-1$ are double space-time indices. Therefore, the $O(D, D)$ metric $\eta_{M N}$ has the following off-diagonal form

$$
\eta_{M N}=\left(\begin{array}{cc}
0 & 1_{D} \\
1_{D} & 0
\end{array}\right),
$$

where $1_{D}$ is the $D \times D$ identity matrix. Note that $\eta_{M N}$ is invariant under ordinary diffemorphisms. Defining

$$
\eta_{A B}=\eta\left(E_{A}, E_{B}\right)
$$

\footnotetext{
${ }^{9}$ Here we are fixing a "diagonal" gauge, by choosing a single frame $e_{a}$ (as opposed to two, as originally in $[8,9]$ ) for the tangent bundle. For more general expressions using two different frames see for example [26].

${ }^{10} \iota_{v}$ is the contraction along the vector $v$ (on a one-form, this is $\iota_{v} \xi=v^{m} \xi_{m}$ ).
} 
where $A, B=0,1, \ldots, 2 D-1$ are frame indices, it is easy to see that when the frame $E_{A}$ is of the form (4.4), $\eta_{A B}$ has also the off-diagonal form

$$
\eta_{A B}=\left(\begin{array}{cc}
0 & 1_{D} \\
1_{D} & 0
\end{array}\right)
$$

One can alternatively use a right-left basis $\mathcal{C}$ by rotating the frame indices with

$$
R_{A}{ }^{B}=\frac{1}{\sqrt{2}}\left(\begin{array}{cc}
1 & -1 \\
1 & 1
\end{array}\right),
$$

namely, $E_{A} \rightarrow\left(E_{\mathcal{C}}\right)_{A}=R_{A}^{B} E_{B}$. In this basis $\eta$ becomes diagonal

$$
\left(R \eta R^{T}\right)_{A B}=\left(\begin{array}{cc}
-1_{D} & 0 \\
0 & 1_{D}
\end{array}\right) .
$$

As in ordinary geometry, the generalized tangent bundle admits a generalized metric defined as

$$
H=\mathcal{S}^{A B} E_{A} \otimes E_{B},
$$

where $\mathcal{S}^{A B}=\operatorname{diag}\left(s^{a b}, s_{a b}\right), s_{a b}$ being the Minkowski metric.

In DFT, the generalized tangent bundle would correspond to the tangent bundle of a double space which locally is parametrized with a double set of coordinates $(x, \tilde{x})$, in which also the derivatives $\partial$ belong to the fundamental representation of $O(D, D)$. The generalized vectors transform under generalized diffeomorphisms as

$$
\mathcal{L}_{V} W^{M}=V^{P} \partial_{P} W^{M}+\left(\partial^{M} V_{P}-\partial_{P} V^{M}\right) W^{P} .
$$

The algebra of generalized diffeomorphisms closes provided a set of constraints is satisfied. From the level matching condition $L_{0}-\bar{L}_{0}=0$ (2.7), recasted as the eigenvalue equation (2.20), extended to all coordinates and restricted to the zero mode $N=\bar{N}=0$, one derives the so-called weak constraint

$$
\partial_{M} \partial^{M} \cdots=0
$$

which holds on a non-compact space. The dots represent fields and/or gauge parameters. On a compact space the weak constraint reads

$$
\partial_{M} \partial^{M} \cdots=-p . \tilde{p} \cdots=(N-\bar{N}) \cdots .
$$

Since this constraint is not enough (the product of fields does not even satisfies it), the strong constraint or section condition is required, in the non compact case and with $N=\bar{N}=0$.

$$
\partial_{M} \cdots \partial^{M} \cdots=0 .
$$

This condition is sufficient to satisfy the closure constraints, but there are more general solutions [27, 29] (see also [41]), as we shall discus in section 4.4. Note that here we do 
not restrict to the zero mode, however the weak constraint on the compact space is never violated, as we will discuss in detail in section 4.4.

One can also incorporate the dilaton $\varphi$ as part of the fields. It is contained in a density field $e^{-2 d}=\sqrt{|g|} e^{-2 \varphi}$ which transforms like a measure

$$
\mathcal{L}_{V} e^{-2 d}=\partial_{P}\left(V^{P} e^{-2 d}\right) .
$$

The generalized diffeomorphisms allow to define the generalized fluxes ${ }^{11}[8,9]$

$$
\mathcal{F}_{A B C}=\left(\mathcal{L}_{E_{A}} E_{B}\right)^{M} E_{C M} .
$$

These are totally antisymmetric in $A B C$ (flat indices) and transform as scalars under generalized diffeomorphisms, up to the closure constraints. The latter become Bianchi identities when the strong constraint is imposed.

In generalized Scherk-Schwarz compactifications [19, 20] one splits the frame into a piece involving the external $d$-dimensional coordinates $x$ and a piece that strictly depends on the internal $n$-dimensional (where $D=d+n$ ) ones $y$, and possibly their duals $\tilde{y}$, namely

$$
E_{A}(x, y, \tilde{y})=\mathcal{U}_{A}{ }^{A^{\prime}}(x) E_{A^{\prime}}^{\prime}(y, \tilde{y}) .
$$

The matrix $\mathcal{U}$ gives account of the fields in the effective theory, while $E^{\prime}$ is a generalized frame that depends on the internal coordinates. All the dependence on the internal coordinates is through the frame. We will discuss this in more detail later. Doing this split, one gets

$$
H=\mathcal{S}^{A B} \mathcal{U}_{A} A^{\prime} E_{A^{\prime}}^{\prime} \mathcal{U}_{B}^{B^{\prime}} E_{B^{\prime}}^{\prime}=\mathcal{H}^{A^{\prime} B^{\prime}} E_{A^{\prime}}^{\prime} E_{B^{\prime}}^{\prime}
$$

where we have defined

$$
\mathcal{H}^{A^{\prime} B^{\prime}}(x)=\mathcal{S}^{A B} \mathcal{U}_{A} A^{\prime} \mathcal{U}_{B}^{B^{\prime}}
$$

As mentioned, the fields of the reduced theory are encoded in $\mathcal{U}$ or directly one can think of $\mathcal{H}$ as parametrizing the moduli space. We will be particularly interested on the "internal piece" of the generalized metric $\mathcal{H}^{I J}$, where $I, J=1, \ldots, 2 n$ are frame indices on the internal part of the double tangent space. Since the internal piece of $H$ lies in $O(n, n) / O(n) \times O(n)$, it can be parametrized by an $n \times n$ matrix whose elements can be written in terms of symmetric and antisymmetric fields denoted $h$ and $b$, respectively.

$$
\mathcal{H}^{I J}=\left(\begin{array}{cc}
h^{-1} & -h^{-1} b \\
b h^{-1} & h-b h^{-1} b
\end{array}\right) .
$$

Note that this is a parametrization of the scalar fields, and they are not the metric and B fields in the internal space (the latter are rather encoded in $E^{\prime}$ ). When $h=1_{n}$ and $b=0$, which we will call "the origin of moduli space", $\mathcal{H}$ reduces to $1_{2 n}$.

In the rotated basis, $\mathcal{H}_{\mathcal{C}}^{I J}$ reads

$$
\mathcal{H}_{\mathcal{C}}=\left(R \mathcal{H} R^{T}\right)=\left(\begin{array}{ll}
\mathcal{H}^{11} & \mathcal{H}^{12} \\
\mathcal{H}^{21} & \mathcal{H}^{11}
\end{array}\right),
$$

\footnotetext{
${ }^{11}$ Additionally, a DFT formulation in terms of fluxes [19, 20,27], requires the generalized fluxes $\mathcal{F}_{A}=$ $e^{-2 d} \mathcal{L}_{E_{A}} e^{2 d}$.
} 
with

$$
\begin{aligned}
\mathcal{H}^{11} & =\left(\mathcal{H}^{22}\right)^{T}=\frac{1}{2}\left[\left(h+h^{-1}\right)+\left(h^{-1} b-b h^{-1}\right)-b h^{-1} b\right], \\
\mathcal{H}^{12} & =\left(\mathcal{H}^{21}\right)^{T}=-\frac{1}{2}\left[\left(h-h^{-1}\right)+\left(h^{-1} b+b h^{-1}\right)-b h^{-1} b\right] .
\end{aligned}
$$

In analogy with (4.17), one defines the internal fluxes $f_{I J}{ }^{K}$ by ${ }^{12,13}$

$$
\left[E_{I}^{\prime}, E_{J}^{\prime}\right]=\mathcal{L}_{E_{I}^{\prime}} E_{J}^{\prime}=f_{I J}{ }^{K} E_{K}^{\prime}
$$

For the generalised Scherk-Schwarz reduction, these have to be constant and satisfy the constraints

$$
f_{I J K} \equiv \eta_{K L} f_{I J}^{L}=f_{[I J K]}, \quad f_{[I J}^{L} f_{K] L}^{R}=0 .
$$

All the information about the internal space is encoded in the structure constants. In the next section we discuss in detail the reduction on a (double) circle, and then we extend this to account for the symmetry enhancement at the self-dual radius.

\subsection{Circle reduction}

In this section we reduce the generalized frame and its corresponding generalized metric on a circle, to set the starting point for the enhancement to be discussed in the next section.

Here we use the following notation for the indices: $\hat{\mu}, \hat{\nu}, \cdots=0, \ldots, d$ label the $D=$ $d+1$-dimensional spacetime indices and $\hat{a}, \hat{b} \ldots$ are their frame index counterparts. To lighten the notation, the coordinate on the circle, previously denoted $Y$, will be called $y$ from now on, and its left and right-moving components $y^{L}, y^{R}$.

We start from a generalized frame of the form (4.4) in $d+1$ dimensions and split it into $d$ non-compact directions and the circle direction $y$. Vectors split as $v^{\hat{\mu}}=\left(v^{\mu}, v^{d}\right)$ with $\mu=0, \ldots, d-1$, the coordinates $x^{\hat{\mu}}=\left(x^{\mu}, y\right), y$ labeling the circle, and the frame indices $\hat{a}=\{a, d\}$. Here we make the identification

$$
y \sim y+2 \pi R_{\mathrm{sd}}
$$

The form frame along the circle is

$$
e^{d}=\phi\left(d y+V_{1}\right)
$$

where $V_{1}=V_{\mu} d x^{\mu}$ is a one-form on the base $M_{d}$. This implies that the metric on the circle is

$$
g_{y y}=\phi^{2}(x)
$$

which means that the physical radius of the circle is given by

$$
R=R_{s d}\langle\phi(x)\rangle
$$

\footnotetext{
${ }^{12}$ Note that this is a vectorial equality, where the generalized vectors have $2 n$ components.

${ }^{13}$ Strictly speaking, the commutator, or C-bracket, between two generalized vectors is defined as $[V, W]=$ $\frac{1}{2}\left(\mathcal{L}_{V} W-\mathcal{L}_{W} V\right)$. However on a frame, $\mathcal{L}_{E_{I}} E_{J}$ is automatically antisymmetric in $I$ and $J$.
} 
On the other hand, the scalar field $\phi$ is parametrized in terms of the fluctuation $M_{33}$ as

$$
\phi(x)=\exp \left(M_{33}(x)\right)
$$

in accordance with (3.40). When this field gets a vev $\epsilon$ as in (2.23), which we write here

$$
M_{33}(x)=\epsilon+M_{33}^{\prime}(x),
$$

we recover the $\epsilon$-expansion of $R$ in (3.20).

Let us now construct the generalized frame. After the circle compactification, the ordinary frame splits into

$$
\hat{e}^{\hat{a}}=\left(e^{a}, e^{d}\right),
$$

where $e^{d}$ is defined in (4.27). The dual frame splits as

$$
\hat{e}_{\hat{a}}=\left(\begin{array}{c}
e_{a}-\iota_{e}^{a} V_{1} \partial_{y} \\
\phi^{-1} \partial_{y}
\end{array}\right)
$$

The 2-form field also splits into

$$
\hat{B}_{2}=B_{2}+B_{1} \wedge\left(d y+V_{1}\right),
$$

where $B_{2}$ has no legs along the circle $\left(\iota_{\partial_{y}} B_{2}=0\right)$ and $B_{1}$ is a one-form on the base $\left(B_{1}=B_{\mu} d x^{\mu}\right)$.

Collecting all the pieces together, the generalized frame (4.4) takes the form

$$
\begin{aligned}
& E_{a}=e_{a}-\left(\iota_{e_{a}} V_{1}\right) \partial_{y}-\left(\iota_{e_{a}} B_{1}\right) d y-\iota_{e_{a}}^{\prime} C^{+} \\
& E_{d}=\phi^{-1}\left(\partial_{y}+B_{1}\right) \\
& E^{d}=\phi\left(d y+V_{1}\right) \\
& E^{a}=e^{a}
\end{aligned}
$$

where $\iota^{\prime}$ denotes the contraction in the first component, i.e $\left(\iota_{e_{a}}^{\prime} C^{+}\right)_{\nu}=e_{a}{ }^{\mu} C_{\mu \nu}^{+}$and

$$
C^{+}=\left(B_{2}+V_{1} \wedge B_{1}\right)+V_{1} B_{1} .
$$

Let us concentrate now on the internal components. We have

$$
\left(\begin{array}{l}
E_{d} \\
E^{d}
\end{array}\right)=\left(\begin{array}{cc}
\phi^{-1} & 0 \\
0 & \phi
\end{array}\right)\left(\begin{array}{l}
\partial_{y}+B_{1} \\
d y+V_{1}
\end{array}\right) .
$$

We can perform a rotation in order to write the expressions in terms of left and right sectors, as they appear in section 3.2, where the $O(1,1)$ matrix $\eta$ takes the form (4.10). Using the rotation matrix defined in (4.9) we get

$$
\left(\begin{array}{c}
\bar{E} \\
E
\end{array}\right)=R\left(\begin{array}{cc}
\phi^{-1} & 0 \\
0 & \phi
\end{array}\right) R^{T} R\left(\begin{array}{c}
\partial_{y}+B_{1} \\
d y+V_{1}
\end{array}\right)
$$




$$
=\left(\begin{array}{cc}
U^{+} & -U^{-} \\
-U^{-} & U^{+}
\end{array}\right)\left(\begin{array}{c}
-\sqrt{2} \overline{\mathcal{J}}^{3}-\frac{1}{\sqrt{2}} \bar{A} \\
\sqrt{2} \mathcal{J}^{3}+\frac{1}{\sqrt{2}} A
\end{array}\right)
$$

where we have defined ${ }^{14}$

$$
\begin{aligned}
A & =V_{1}+B_{1}, & \mathcal{J}^{3} & =\frac{1}{2}\left(d y+\partial_{y}\right), \\
\bar{A} & =V_{1}-B_{1}, & \overline{\mathcal{J}}^{3} & =\frac{1}{2}\left(d y-\partial_{y}\right),
\end{aligned}
$$

and

$$
U^{ \pm}=\frac{1}{2}\left(\phi \pm \phi^{-1}\right)
$$

Using the relation between $\phi$ and $M_{33}$ given in (4.30), we get

$$
\begin{aligned}
& U^{+}=\cosh \left(M_{33}\right)=1+\mathcal{O}\left(M_{33}\right)^{2}, \\
& U^{-}=\sinh \left(M_{33}\right)=M_{33}+\mathcal{O}\left(M_{33}\right)^{3}
\end{aligned}
$$

where the expansion holds when $M_{33}^{\prime} \ll 1$ and $\epsilon \ll 1$.

Computing the generalized metric (4.20) in the rotated basis we get

$$
\mathcal{H}_{\mathcal{C}}=\left(\begin{array}{cc}
\cosh \left(2 M_{33}\right) & -\sinh \left(2 M_{33}\right) \\
-\sinh \left(2 M_{33}\right) & \cosh \left(2 M_{33}\right)
\end{array}\right) \approx\left(\begin{array}{cc}
1 & -2 M_{33} \\
-2 M_{33} & 1
\end{array}\right)+\mathcal{O}\left(M_{33}\right)^{2} .
$$

Note that this has precisely the form (4.22) if we identify

$$
M_{33}=h_{0},
$$

where $h_{0}$ is defined as the perturbation of $h$ (in one dimension $b=0$ ),

$$
h \approx 1+2 h_{0} .
$$

Having discussed the scalar fields, which depend on the external coordinates, let us now go back to the frame (4.38), and concentrate only on the piece that depends on the "internal coordinates", encoded in $\mathcal{J}^{3}, \overline{\mathcal{J}}^{3}$. Following the standard procedure in DFT, we identify the vector $\partial_{y}$ with a one-form along the "winding coordinate", or viceversa (the one-form $d y$ with a vector along the dual coordinate) i.e

$$
\partial_{y} \rightarrow d \tilde{y}, \quad \tilde{y} \sim \tilde{y}+2 \pi \tilde{R}_{\mathrm{sd}} .
$$

At this level, we are allowed to formally do that. However, if we introduce a dependence of the fields on $\tilde{y}$ at the same time as a dependence on $y$, we would violate the strong constraint (4.15). For the time being, we are not introducing any explicit dependence on the coordinates, so (4.44) is just a formal relabeling. We will come back to this point in section 4.4. With that relabeling, we have

$$
\mathcal{J}=d y^{L}, \quad \overline{\mathcal{J}}=d y^{R},
$$

\footnotetext{
${ }^{14} \mathrm{As}$ in section 3.2 , a bar indicates a right-moving sector, not complex conjugate.
} 
where, as usual, these come from the splitting

$$
y=y^{L}+y^{R}, \quad \tilde{y}=y^{L}-y^{R} .
$$

Including also the uncompactified directions, the full generalized frame then takes the form

$$
\left(\begin{array}{c}
E_{a} \\
\bar{E} \\
E \\
E^{a}
\end{array}\right)=\left(\begin{array}{cccc}
1_{d} & 0 & 0 & 0 \\
0 & U^{+} & -U^{-} & 0 \\
0 & -U^{-} & U^{+} & 0 \\
0 & 0 & 0 & 1_{d}
\end{array}\right)\left(\begin{array}{c}
e_{a}+\iota_{e_{a}} \bar{A} \overline{\mathcal{J}}^{3}-\iota_{e_{a}} A \mathcal{J}^{3}-\iota_{e_{a}}^{\prime} C^{+} \\
-\sqrt{2} \overline{\mathcal{J}}^{3}-\frac{1}{\sqrt{2}} \bar{A} \\
\sqrt{2} \mathcal{J}^{3}+\frac{1}{\sqrt{2}} A \\
e^{a}
\end{array}\right)
$$

For convenience, here we have written the frame partially in the Scherk-Schwarz form (4.18), in the sense that the first matrix involves only the scalar fields of the reduced theory, while the vector and tensor fields $A, \bar{A}, e_{a}{ }^{\mu}, B_{\mu \nu}$ are contained in the second matrix. Note however that all the latter depend on the external coordinates only. In the case of the circle, giving rise to $\mathrm{U}(1)_{L} \times \mathrm{U}(1)_{R}, \mathcal{J}^{3}$ and $\overline{\mathcal{J}}^{3}$ are constant, (they are given in (4.45)). This will no longer be the case when we describe the enhancement.

In the next section, $\mathcal{J}^{3}$ and $\overline{\mathcal{J}}^{3}$ will be promoted to the $\mathrm{SU}(2)_{L} \times \mathrm{SU}(2)_{R}$ generators, and the elements $U^{ \pm}$in (4.2) to $3 \times 3$ matrices.

\subsection{Extension and enhanced gauge symmetry}

In the previous section we have written the generalized frame and the generalized metric in $O(d+1, d+1)$ form, where we were able to include all the fields of the reduced theory associated to the states that are massless in $d+1$ dimensions. We have also argued that in order to include the extra fields with non-zero compact momentum and/or winding number that are massless at the self-dual radius, or in other words, to account for the enhancement of the gauge group, we need to promote the $O(d+1, d+1)$ symmetry to $O(d+3, d+3)$.

We therefore identify $A, \bar{A}$ with the $\mathrm{SU}(2)_{L} \times \mathrm{SU}(2)_{R}$ components $A^{3}, \bar{A}^{3}$, and promote them to the triplet $A^{i}, \bar{A}^{i}$, and similarly for $\mathcal{J}^{3}, \overline{\mathcal{J}}^{3}$. Promoting the scalar $M_{33}$ to the matrix $M_{i j}$ at the level of the frames is trickier since now, the $6 \times 6$ internal matrix twisting the frame has to be an element of $O(3,3)$, parametrizing the coset $O(3,3) / O(3) \times O(3)$. The extension of the frame (4.47) reads

$$
\left(\begin{array}{c}
E_{a} \\
\bar{E}^{i} \\
E^{i} \\
E^{a}
\end{array}\right)=\left(\begin{array}{cccc}
1_{d} & 0 & 0 & 0 \\
0 & U_{1}^{i j} & U_{2}^{i j} & 0 \\
0 & U_{3}^{i j} & U_{4}^{i j} & 0 \\
0 & 0 & 0 & 1_{d}
\end{array}\right)\left(\begin{array}{c}
e_{a}+\iota_{e} \bar{A}^{k} \overline{\mathcal{J}}^{k}-\iota_{e_{a}} A^{k} \mathcal{J}^{k}-\iota_{e_{a}}^{\prime} C^{+} \\
-\sqrt{2} \overline{\mathcal{J}}^{j}-\frac{1}{\sqrt{2}} \bar{A}^{j} \\
\sqrt{2} \mathcal{J}^{j}+\frac{1}{\sqrt{2}} A^{j} \\
e^{a}
\end{array}\right)
$$

where the six vectors $A^{i}, \bar{A}^{i}$ are those introduced in section 2.1, and the matrices $U$ have a very complicated dependence on the scalars. To see how the scalars $M_{i j}$ fit in the DFT description, it is simpler to look at the $6 \times 6$ "internal" block of the generalized metric (4.22). Noticing that $(4.2)$ is an element of $O(1,1)$ close to the identity, the natural extension is to 
consider an element of $\mathrm{SO}^{+}(3,3) / \mathrm{SO}(3) \times \mathrm{SO}(3), \mathrm{SO}^{+}(3,3)$ being the identity component of $O(3,3)$, near $1_{6}$. Such an element can be generally written as

$$
\mathcal{H}_{\mathcal{C}}=\left(\begin{array}{cc}
1_{3} & -2 M \\
-2 M^{T} & 1_{3}
\end{array}\right)
$$

as can be easily seen by expanding the "auxiliary fields" $h, b$ of (4.23) around the origin of moduli space, namely, by setting

$$
h \approx 1_{3}+2 h_{0}, \quad b \approx 0_{3}+b_{0},
$$

with $h_{0}, b_{0}$ small, and $2 M=2 h_{0}+b_{0}$.

All these facts allow us to think of $M_{i j}$ as parametrizing the scalar fields (which depend on the external coordinates) corresponding to the fluctuations of a metric and a b-field of an "internal three-dimensional space". All the dependence on the "internal coordinates" is hidden in the $\mathcal{J}^{i}, \overline{\mathcal{J}}^{i}$, where we now focus our attention. These generators are such that they satisfy the $\mathrm{SU}(2)_{L} \times \mathrm{SU}(2)_{R}$ algebra

$$
\left[\mathcal{J}^{i}, \mathcal{J}^{j}\right]=\frac{1}{\sqrt{\alpha^{\prime}}} \epsilon^{i j k} \mathcal{J}^{k}, \quad\left[\overline{\mathcal{J}}^{i}, \overline{\mathcal{J}}^{j}\right]=\frac{1}{\sqrt{\alpha^{\prime}}} \epsilon^{i j k} \overline{\mathcal{J}}^{k}, \quad\left[\mathcal{J}^{i}, \overline{\mathcal{J}}^{j}\right]=0
$$

under some bracket. This is all we need to know about the $\mathcal{J}$ 's in order to compute the effective action from DFT. We would like however to find explicit realizations of this algebra, and discuss further this "internal space" or rather "internal double space". This is the subject of the following section.

Before closing this section, let us note that interestingly enough, T-duality can be embedded in $O(3,3)$ through the matrix

$$
\mathcal{D}=\left(\begin{array}{cc}
1_{3} & 0 \\
0 & D
\end{array}\right)
$$

where $D$ is given by (3.19). Indeed, it can be straightforwardly checked that

$$
\mathcal{D}^{T} \mathcal{H}_{\mathcal{C}} \mathcal{D}=\left(\begin{array}{cc}
1_{3} & -2 M D \\
-2(M D)^{T} & 1_{3}
\end{array}\right),
$$

as expected. The recognition of T-duality as a part of the enhanced gauge symmetry relies, within this context, on the fact that $\mathcal{D}$ belongs to $O(3) \times O(3)$.

\subsection{Internal double space}

Here we discuss explicit realizations of the $\mathrm{SU}(2)_{L} \times \mathrm{SU}(2)_{R}$ algebra (4.51), and its corresponding "internal space".

In the bosonic string compactified on a circle, the $D$-dimensional manifold is a $\mathrm{U}(1)$ fibration with connection $V_{1}$ over a $D-1=d$ dimensional base (the uncompactified external space). The B-field with one leg along the fiber contributes to a second one-form, and with both of them one builds $A, \bar{A}$ as the gauge bosons of a $\mathrm{U}(1)_{L} \times \mathrm{U}(1)_{R}$ symmetry. 
At the self-dual radius, extra vectors that have non-zero discrete momentum and winding number become massless, $A^{ \pm}, \bar{A}^{ \pm}$. The way we have incorporated them in the DFT/GCG geometry description is by extending the generalized tangent space such that it transforms in the fundamental representation of $O(D+2, D+2)=O(d+3, d+3)$. One can think of the four extra vectors $A^{ \pm}, \bar{A}^{ \pm}$as coming from "a metric and a B-field" with one leg along these "extra two directions". This is just a book-keeping device, the ordinary space is still $D$ dimensional, and (so-far) we allow dependence only on the coordinate on the circle, $y$. However, if we only allow dependence on a single internal coordinate $y$, there is no way of realizing the $\mathrm{SU}(2)_{L} \times \mathrm{SU}(2)_{R}$ algebra. At the same time, the philosophy of DFT is that in order to have a field theory for strings, where one can have not only momentum but also winding modes, one needs to incorporate the coordinates dual to winding. We therefore introduce the coordinate $\tilde{y}$ as in (4.44), and allow for explicit dependence on $y, \tilde{y}$. Once we do that, the identification (4.44) is not formal anymore (i.e. in the generalized diffeomorphisms (4.12), the derivative $\partial_{M}$ will have both components $\partial_{y}$ and $\partial_{\tilde{y}}$ that will act non-trivially). We know however that by allowing dependence on $y$ and $\tilde{y}$ we might violate the strong constraints (4.14), (4.15). Violating the former is expected since we do not restrict to the zero mode of the eigenvalue equation (2.20). Nevertheless, within the class of configurations of the generalized Scherk-Schwarz type $(4.18)^{15}$ where all the dependence on $y, \tilde{y}$ is fully encoded in the frame $E^{\prime}$, the theory is consistent at the classical level as long as the gaugings (4.24) coming from the frame $E^{\prime}$ satisfy the quadratic constraints of gauged supergravity (equation on the right of (4.25)) [29]. These are weaker than the strong constraint, and allow in our case to incorporate the momentum and winding modes.

To recap, the idea here is that we have a $d+1+1$ dimensional manifold of the form

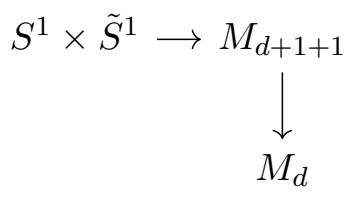

Over this manifold, we define a generalized tangent bundle that is locally

$$
E \simeq T M_{d} \oplus V_{2} \oplus T S^{1} \oplus T \tilde{S}^{1} \oplus V_{2}^{*} \oplus T^{*} M_{d}
$$

where $V_{2}$ is a two-dimensional vector bundle (and $V_{2}^{*}$ its dual) that we add to accommodate the extra massless gauge vectors and scalars. Let $\left(e_{1}, e_{2}\right)$ be a global frame for $V_{2}$ (with dual frame $e^{1}, e^{2}$ ), and build the corresponding basis of left-moving and right-moving one-forms $e^{1 L(R)}, e^{2 L(R)}$.

The following frame on $E[33]$

$$
\begin{aligned}
& \overline{\mathcal{J}}^{1}=\cos \left(2 y^{R} / \sqrt{\alpha^{\prime}}\right) e^{1 R}+\sin \left(2 y^{R} / \sqrt{\alpha^{\prime}}\right) e^{2 R} \\
& \overline{\mathcal{J}}^{2}=-\sin \left(2 y^{R} / \sqrt{\alpha^{\prime}}\right) e^{1 R}+\cos \left(2 y^{R} / \sqrt{\alpha^{\prime}}\right) e^{2 R} \\
& \overline{\mathcal{J}}^{3}=d y^{R}
\end{aligned}
$$

\footnotetext{
${ }^{15}$ Within this class, there is no distinction between the weak and the strong constraint, one implies the other [29].
} 


$$
\begin{aligned}
& \mathcal{J}^{1}=\cos \left(2 y^{L} / \sqrt{\alpha^{\prime}}\right) e^{1 L}+\sin \left(2 y^{L} / \sqrt{\alpha^{\prime}}\right) e^{2 L} \\
& \mathcal{J}^{2}=-\sin \left(2 y^{L} / \sqrt{\alpha^{\prime}}\right) e^{1 L}+\cos \left(2 y^{L} / \sqrt{\alpha^{\prime}}\right) e^{2 L} \\
& \mathcal{J}^{3}=d y^{L}
\end{aligned}
$$

realizes, under the C-bracket of generalized diffeomorphisms (4.24), the $\mathrm{SU}(2)_{L} \times \mathrm{SU}(2)_{R}$ algebra (4.51). To derive this, we use the fact that in the generalised diffemorphisms in (4.24), (4.12) the derivative has non-trivial components

$$
\partial_{P}=\left(0,0, \partial_{y^{R}}, 0,0, \partial_{y^{L}}\right)
$$

and its indices are raised with

$$
\eta^{P Q}=\left(\begin{array}{cc}
-1_{3} & 0 \\
0 & 1_{3}
\end{array}\right) .
$$

These are precisely the CFT current operators $J^{i}$ in (2.13) (up to factors from using slightly different conventions $)^{16}$ provided the complex combinations of $e^{i}$ project on the worldsheet as $d z$ or $d \bar{z}$. To be more precise, we have

$$
\begin{array}{lll}
\overline{\mathcal{J}}^{ \pm}=e^{\mp \frac{2 i}{\sqrt{\alpha^{\prime}}} y^{R}} e^{ \pm R} \leftrightarrow e^{\mp \frac{2 i}{\sqrt{\alpha^{\prime}}} y^{R}(\bar{z})} d \bar{z}=\bar{J}^{\mp} d \bar{z}, & \overline{\mathcal{J}}^{3}=i / \sqrt{\alpha^{\prime}} d y^{R} \leftrightarrow d y^{R}(\bar{z})=\bar{J}^{3} d \bar{z} \\
\mathcal{J}^{ \pm}=e^{\mp \frac{2 i}{\sqrt{\alpha^{\prime}}} y^{L}} e^{ \pm L} \leftrightarrow e^{\mp \frac{2 i}{\sqrt{\alpha^{\prime}}} y^{L}(z)} d z=J^{\mp} d z, & \mathcal{J}^{3}=i / \sqrt{\alpha^{\prime}} d y^{L} \leftrightarrow d y^{L}(z)=J^{3} d z .
\end{array}
$$

We emphasize once more that the frame (4.55) violates the strong constraint, namely it depends on $y^{L}$ and $y^{R}$, or equivalently on $y$ and $\tilde{y}$. In other words it is "non-geometric". Nevertheless, the algebra satisfies the quadratic constraints (4.25), and therefore the generalized Scherk-Schwarz reduction based on this frame is consistent. Note however something very interesting, which is the same that happens when realizing the new $\mathrm{SO}(8)$ gaugings of [35], as discussed in [34]: if we compute the generalized metric (4.11) for the frame $E^{\prime}$, we get

$$
\mathcal{H}^{\prime}\left(y^{L}, y^{R}\right)=\delta^{I J} E_{I}^{\prime} \otimes E_{J}^{\prime}=2 \mathbb{I}_{6},
$$

i.e. all the dependence on $y^{L}, y^{R}$ drops out. This implies that what is "non-geometric" is the frame, not the space. Since in Scherk-Schwarz compactifications one keeps the modes associated to the frame, these carry the information about the "non-geometric gaugings". One could have thought of alternatively taking the frame given by $e^{1 L}, e^{2 L}$ instead of $E_{1}^{\prime}, E_{2}^{\prime}$ (and similarly for the right sector), which are also globally defined. However, if we had done a generalized Scherk-Schwarz reduction based on this frame, we would have precisely missed the modes with non-zero momentum and winding along the circle. Furthermore, if we had taken this frame, we would have included modes with non-zero left and right-moving oscillator numbers $N_{i}$ along $i=1,2$, which does not make sense, since these directions are

\footnotetext{
${ }^{16}$ Recall that the well defined conformally invariant vertex operator to be integrated on the world-sheet is of the form $V(z, \bar{z}) d z d \bar{z}$ where $V(z, \bar{z})$ is one of the vertex operators defined above. Therefore, the currents will always appear as $J(z) d z$ (and $\bar{J}(\bar{z}) d \bar{z}$ ) namely as world-sheet one forms. Also while $J^{3}(z) d z \sim d y(z)$ can be interpreted as the pullback of an exact one-form on the internal space time, the other currents, related to winding states, cannot.
} 
fictitious and the string cannot have an oscillation number along them. A more detailed discussion of this for (ordinary) Scherk-Schwarz reductions can be found in [36].

Another way of understanding this point is the following [34]: while all the dependence on the internal doubled coordinates drops out in $\mathcal{H}^{\prime}$ as defined in (4.58), in the full generalized metric $H$ of (4.19), which is based on the frame $E(x, y)$, generically it will not. Namely

$$
\mathcal{H}=\left(U E^{\prime}\right)^{T}\left(U E^{\prime}\right)=\left(\begin{array}{cc}
1_{3} & -R_{L}^{T} 2 M R_{R} \\
-R_{R}^{T} 2 M^{T} R_{L} & 1_{3}
\end{array}\right)
$$

where $R_{L}$ is the rotation matrix

$$
R_{L}=\left(\begin{array}{ccc}
\cos \left(2 y^{L} / \sqrt{\alpha^{\prime}}\right) & \sin \left(2 y^{L} / \sqrt{\alpha^{\prime}}\right) & 0 \\
-\sin \left(2 y^{L} / \sqrt{\alpha^{\prime}}\right) & \cos \left(2 y^{L} / \sqrt{\alpha^{\prime}}\right) & 0 \\
0 & 0 & 1
\end{array}\right)
$$

used to construct the frame $E^{\prime}$ in (4.55), and similarly for the right sector. We see that only when the fluctuations $M$ are turned off (or rather, only when all fluctuations but $M_{33}$ are turned off, which corresponds with the ordinary KK reduction on $S^{1}$ ), the dependence on $y^{L}$ and $y^{R}$ (or analogously $y$ and $\tilde{y}$ ) drops out from the generalized metric. Whenever this is not the case, i.e., when one turns on the fields of the low energy theory, or in other words one considers fluctuations around the background, the dependence on $y$ and $\tilde{y}$ remains. This implies that, while the background (no fluctuations) is geometric, the fluctuations are not.

\subsection{Effective action from DFT}

To obtain the effective $d$-dimensional action, we start from the $O(D, D)$ action (where for us $D=d+3)[3-5]$

$$
S=\frac{1}{2 \kappa_{d+3}^{2}} \int d X e^{-2 d}[\mathbb{R}(\mathcal{H}, d)-\Lambda]
$$

with

$$
\begin{aligned}
\mathbb{R}= & 4 \mathcal{H}^{M N} \partial_{M} \partial_{M} d-\partial_{M N} \mathcal{H}^{M N}-4 \mathcal{H}^{M N} \partial_{M} d \partial_{N} d+4 \partial_{M} \mathcal{H}^{M N} \partial_{N} d \\
& +\frac{1}{8} \mathcal{H}^{M N} \partial_{M} \mathcal{H}^{K L} \partial_{N} \mathcal{H}_{K L}-\frac{1}{2} \mathcal{H}^{M N} \partial_{M} \mathcal{H}^{K L} \partial_{K} \mathcal{H}_{N L}
\end{aligned}
$$

the generalized Ricci scalar [31] and $\Lambda$ a manifestly $O(D, D)$ invariant cosmological constant term that can be added to the action of DFT [32]. The reason for including this term will become clear shortly. On the $d$-dimensional external space we use the strong constraint and let fields depend only on the usual space-time coordinates $x$, while regarding the internal piece, we let the derivative $\partial_{M}$ act non trivially on both variables, $y^{L}$ and $y^{R}$.

Performing a generalized Scherk-Schwarz reduction of this action of the form (4.18), and integrating over the $D-d$ "double internal space", the $d$-dimensional effective action has been obtained in $[19,20]$. It reads

$$
S_{\mathrm{eff}}=\frac{1}{2 \kappa_{d}^{2}} \int d^{d} x \sqrt{g} e^{-2 \varphi}\left[\mathcal{R}+4 \partial^{\mu} \varphi \partial_{\mu} \varphi-\frac{1}{12} H_{\mu \nu \rho} H^{\mu \nu \rho}\right.
$$




$$
\begin{gathered}
-\frac{1}{8} \mathcal{H}_{I J} F^{I \mu \nu} F_{\mu \nu}^{J}+\frac{1}{8}\left(D_{\mu} \mathcal{H}\right)_{I J}\left(D^{\mu} \mathcal{H}\right)^{I J} \\
\left.-\frac{1}{12} f_{I J}{ }^{K} f_{L M}{ }^{N}\left(\mathcal{H}^{I L} \mathcal{H}^{J M} \mathcal{H}_{K N}-3 \mathcal{H}^{I L} \eta^{J M} \eta_{K N}+2 \eta^{I L} \eta^{J M} \eta_{K N}\right)-\Lambda\right] .
\end{gathered}
$$

In this expression $\mathcal{H}_{I J}$ with $I, J=1, \ldots, 2 n$ is the generalized metric containing the scalar fields coming from the internal components of the $n$-dimensional metric and $B$-field, defined in (4.20), $\mathcal{R}$ is the $d$-dimensional Ricci scalar, the field strengths $F_{\mu \nu}^{A}$ and $H_{\mu \nu \rho}$ are

$$
\begin{aligned}
F^{I} & =d A^{I}+\frac{1}{\sqrt{2}} f_{J K}{ }^{I} A^{J} \wedge A^{K} \\
H & =d B+F^{I} \wedge A_{I},
\end{aligned}
$$

and $A^{I}$ are the mixed external-internal components of the $\mathcal{U}$ piece of the frame (the piece that depends on the external coordinates, see (4.18)). The covariant derivative of the scalars is

$$
\left(D_{\mu} \mathcal{H}\right)_{I J}=\left(\partial_{\mu} \mathcal{H}\right)_{I J}+\frac{1}{\sqrt{2}} f^{K}{ }_{L I} A_{\mu}^{L} \mathcal{H}_{K J}+\frac{1}{\sqrt{2}} f^{K}{ }_{L J} A_{\mu}^{L} \mathcal{H}_{I K}
$$

and the generalized fluxes are obtained from the bracket of the $E^{\prime}$ piece of the frame (the part depending on the internal coordinates), as given in (4.24).

Let us now specialize to our case, where $D=d+3$. The fields that depend on the external coordinates, which are the fields of the reduced action, are encoded in the matrix $\mathcal{U}$. This reads (cf. (4.48)),

$$
\mathcal{U}=\left(\begin{array}{cccc}
e_{a} & \iota_{e_{a}} \bar{A}^{k} & -\iota_{e_{a}} A^{k} & -\iota_{e_{a}} C^{+} \\
0 & -\sqrt{2} U_{1}{ }^{i k} & \sqrt{2} U_{2}{ }^{i k} & -\frac{1}{\sqrt{2}}\left(U_{1}^{i j} \bar{A}^{j}-U_{2}{ }^{i j} A^{j}\right) \\
0 & -\sqrt{2} U_{3}{ }^{i k} & \sqrt{2} U_{4}{ }^{i k} & -\frac{1}{\sqrt{2}}\left(U_{3}{ }^{i j} \bar{A}^{j}-U_{4}{ }^{i j} A^{j}\right) \\
0 & 0 & 0 & e^{a}
\end{array}\right)
$$

which implies that the gauge fields are $A_{\mu}^{I}=\left(-\bar{A}_{\mu}^{i}, A_{\mu}^{i}\right)$, and $\mathcal{H}_{I J}$ is as in (4.49). ${ }^{17}$ The internal piece of the frame is given by

$$
\bar{E}^{\prime i}=-\sqrt{2} \overline{\mathcal{J}}^{i}, \quad E^{\prime i}=\sqrt{2} \mathcal{J}^{i},
$$

where $\mathcal{J}^{i}, \overline{\mathcal{J}}^{i}$ realize the $\mathrm{SU}(2)_{L} \times \mathrm{SU}(2)_{R}$ algebra $^{18}$ (4.51). This implies that for us the structure constants in (4.63)-(4.65) are

$$
f_{I J}{ }^{K}=\left\{\begin{array}{c}
-\left(\frac{2}{\alpha^{\prime}}\right)^{\frac{1}{2}} \bar{\epsilon}_{i j k} \\
\left(\frac{2}{\alpha^{\prime}}\right)^{\frac{1}{2}} \epsilon_{i j k}
\end{array} .\right.
$$

\footnotetext{
${ }^{17}$ In $\mathcal{H}_{I J}$ we only need to keep up to terms linear in $M$. Higher order terms give contributions to the effective action that are of order $M^{4}$ and higher, which cannot be compared with the effective string theory action obtained from the three-point functions that we have computed.

${ }^{18}$ Here we do not need to know the way in which the algebra is realized. It could be in the way presented in the previous section, or in whatever other way one may come up with, like for example by a compactification on an $S^{3}$ with $\mathrm{H}$ flux [37, 38], or a compactification on $S_{L}^{3} \times S_{R}^{3}$, under an ordinary 6-dimensional Lie bracket [39].
} 
Inserting all this in (4.63) we get

$$
\begin{gathered}
S=\frac{1}{2 \kappa_{d}^{2}} \int d^{d} x \sqrt{g} e^{-2 \varphi}\left(\mathcal{R}+4 \partial^{\mu} \varphi \partial_{\mu} \varphi-\frac{1}{12} H_{\mu \nu \rho} H^{\mu \nu \rho}-\frac{1}{8}\left(\delta_{i j} F^{i \mu \nu} F_{\mu \nu}^{j}+\delta_{i j} \bar{F}^{i \mu \nu} \bar{F}_{\mu \nu}^{j}\right)\right. \\
\left.-\frac{1}{2} M_{i j} F^{i \mu \nu} \bar{F}_{\mu \nu}^{j}-D_{\mu} M_{i j} D^{\mu} M_{i j}+\frac{16}{\alpha^{\prime}} \operatorname{det} M+\frac{4}{\alpha^{\prime}}-\Lambda\right)+\mathcal{O}\left(M^{4}\right)
\end{gathered}
$$

This is precisely the effective action (2.21), obtained from the string three-point functions at the self-dual radius, if

$$
\Lambda=\frac{4}{\alpha^{\prime}} .
$$

Let us make a few comments about the cosmological constant $\Lambda$ required to match the DFT action with the effective action from string theory. Notice that on the background that we are expanding around, namely

$$
g_{\mu \nu}=\eta_{\mu \nu} ; \quad A_{\mu}=\bar{A}_{\mu}=M_{i j}=0, \quad \varphi=\text { constant },
$$

the condition (4.69) is the equation of motion for the dilaton field. It is then clear that without the addition of the cosmological constant $\Lambda$ to the action it would not be possible to solve the dilaton field equation.

Notice, the same contribution $\frac{4}{\alpha^{\prime}}$ appears in the effective action of the bosonic string on the background metric of an $\mathrm{SU}(2)$ group manifold, corresponding to a sphere $S^{3}$. As is well known, conformal invariance of the two dimensional action requires the addition of a Wess-Zumino term and the relation $R^{2}=k \alpha^{\prime}$ between the radius of the sphere $R$ and the level of the Kac-Moody algebra $k$ [42]. The contributions to the conformal anomaly from the curvature of the compactified space $\left(\frac{6}{R^{2}}\right)$ and from the flux of the antisymmetric tensor field $\left(-\frac{2}{R^{2}}\right)$ in this model add up to $\frac{4}{\alpha^{\prime}}$ at the self-dual radius for $k=1$.

However, our effective internal background is $S^{1} \times \tilde{S}^{1}$, which topologically allows winding states in string theory, unlike the $S^{3}$. Over this $S^{1} \times \tilde{S}^{1}$, we constructed a globally well defined frame (4.55) depending on only two coordinates (the ordinary compact coordinate and the dual one). No more coordinates are needed in order to realize the $\mathrm{SU}(2)_{L} \times \mathrm{SU}(2)_{R}$ algebra, in agreement with the contribution of the Sugawara central charge $c=3-\frac{6}{k+2}$ to the bosonic string critical dimension $d=26-c$ [43], and with the fact that we are describing a critical string with one compact dimension on a circle of self-dual radius. ${ }^{19}$

\section{Conclusions}

We have obtained the effective action for the closed bosonic string compactified on a circle of radius $R$ close to the self-dual point, and reproduced it using the framework of double field theory.

Even if several aspects of the string effective action are well known, an explicit computation, from string amplitudes away from the self dual point, does not seem to be available in the literature. This computation allows for a correct identification of the massive fields,

\footnotetext{
${ }^{19}$ This issue is discussed in a similar context in [44].
} 
which is crucial to get non-anomalous results. The explicit dependence of the effective action on the radius $R$ and its dual $\tilde{R}=\alpha^{\prime} / R$ favors a discussion on the manifestation of T-duality in the field theory. Moreover, the built in Higgs mechanism can be identified in the sense that, very close to the self dual radius, the results can be obtained as coming from a Higgs mechanism in field theory.

In the double field theory calculation, three key steps were involved. The first one required the introduction of a coordinate $\tilde{y}$, T-dual to the circle coordinate and Fourier transform of the winding, as dictated by double field theory. The second one needed an extension of the generalized tangent space with four (two left and two right) additional directions, such that generalized vectors transform in the fundamental representation of $O(d+3, d+3)$ (where $d$ is the number of non-compact directions). These extra directions accommodate the extra states that become massless at the self-dual point. The appearance of the symmetry group $O(d+3, d+3)$ is at the same time natural and intriguing, and might hint at a larger underlying structure encompassing all modes that become massless at given points in moduli space. The third step involved violating the strong constraint by including dependence of the fields on the coordinate of the dual circle. A violation of the strong constraint is expected, as the latter arises from the level matching condition in the absence of momentum together with (non-orthogonal) winding modes, contrary to the situation here.

The effective action was obtained from a generalized Scherk-Schwarz reduction where the frame splits into a piece involving the fields of the reduced theory (the graviton and antisymmetric tensor fields, together with the six vector and nine scalar fields proper to the reduction), and a "twist" that depends on the internal coordinates. The latter depends only on the circle and T-dual circle coordinates, and is such that it reproduces the $\mathrm{SU}(2)_{L} \times \mathrm{SU}(2)_{R}$ algebra under the C-bracket.

This work fills a gap in double field theory: even though the idea of doubling the coordinates originates from an attempt to describe winding modes, to the best of our knowledge the latter had never been clearly included.

At the same time, new questions and avenues to explore open up. One interesting question that is worth understanding better is whether this particular DFT construction, based on the specific kind of enhancement and modes that one is trying to reproduce, is generalizable and can be adapted to other cases (such as for example higher dimensions of the compact space), and how much can be anticipated by general arguments. Another one is the possibility of going to higher orders in $\epsilon$, the parameter measuring the deviation from the self-dual point. Moreover, while in this article we concentrate in gauge symmetry enhancement in the bosonic case, the supersymmetric extension is an open and crucial issue to be explored. Furthermore, we think it is definitely worth investigating whether some or all gaugings in the lower dimensional gauged supergravity that are believed to arise from so-called non-geometric fluxes can be realised in string theory this way.

\section{Acknowledgments}

We thank E. Andrés, P. Cámara, C. Strickland-Constable, G. Torroba, D. Waldram and specially D. Marques for useful discussions and comments. This work was partially sup- 
ported by EPLANET, CONICET, PICT-2012-513 and the ERC Starting Independent Researcher Grant 259133-ObservableString. G. A. thanks the Instituto de Fisica Teorica (IFT UAM-CSIC) in Madrid for its support via the Centro de Excelencia Severo Ochoa Program under Grant SEV-2012-0249. G.A. and M.G. are grateful to the Mainz Institute for Theoretical Physics (MITP) and C.N. and A.R thank the A.S.ICTP for hospitality and partial support during the completion of this work.

\section{A Three-point amplitudes}

The closed string three-point amplitudes are the products of holomorphic and antiholomorphic pieces. To compute amplitudes involving vector and scalar fields, we need the expectation values of two and three currents. The OPE gives

$$
\begin{aligned}
\left\langle J^{i}\left(z_{1}\right) J^{j}\left(z_{2}\right)\right\rangle & =\frac{\delta^{i j}}{2 z_{12}^{2}}, \\
\left\langle J^{i}\left(z_{1}\right) J^{j}\left(z_{2}\right) J^{k}\left(z_{3}\right)\right\rangle & =\frac{i \epsilon^{i j k}}{2\left(z_{12} z_{23} z_{13}\right)} .
\end{aligned}
$$

Similar expressions hold for the antiholomorphic currents. Here the algebra read $\left[t^{i}, t^{j}\right]=$ $i \epsilon^{i j k} t^{k}$ and $\epsilon^{i j k}$ is the Levi-Civita simbol and $i, j, k=1,2,3, \mathrm{SU}(2)$ indices. Amplitudes are evaluated using the mass-shell condition $K_{i}^{2}=-M_{i}^{2}$.

We show general expressions that can be used to obtain the results both at the self-dual radius as well as for the broken symmetry case $m_{-} \neq 0$.

Away from the self-dual radius the complex base corresponding to indices $i=3,+,-$ appears more convenient since fields in this base have well defined charge with repect to the group $U_{L}(1) \times U_{L}(1)_{R}$. Some of the results below are presented in this base. As expected, charges $q_{\phi}, \bar{q}_{\phi}$ of a given field $\phi$ appear in the amplitudes.

The enhanced symmetry expressions are easily obtained by setting $m_{-}=0, \frac{\sqrt{\alpha^{\prime}} m_{+}}{2}=1$ and all charges $q=1$.

Also a gauge condition $\epsilon_{i}^{\prime} \cdot K_{i}=0$ must be used with the effective polarization $\epsilon_{\mu}^{\prime \pm}=\epsilon_{\mu}^{ \pm}+$ $( \pm) \xi K_{\mu} \frac{1}{m_{-}} \phi_{ \pm 3}$ discussed above (3.11). It reduces to the massless vector field transversality condition at the self-dual radius.

A conservation factor $\delta_{k}:=(2 \pi)^{d} \delta^{d}\left(\sum_{i} K_{i}\right)$ is assumed to be present in all expressions.

- Three neutral bosons

$$
\begin{aligned}
\left\langle V_{G} V_{G} V_{G}\right\rangle= & \frac{\pi g_{c}^{\prime}}{2}\left(\left(K_{1} \cdot \epsilon_{3}^{G} \cdot K_{1}\right) \operatorname{Tr}\left(\epsilon_{1}^{G} \cdot \epsilon_{2}^{G}\right)+\left(K_{2} \cdot \epsilon_{1}^{G} \cdot K_{2}\right) \operatorname{Tr}\left(\epsilon_{2}^{G} \cdot \epsilon_{3}^{G}\right)\right. \\
& +\left(K_{3} \cdot \epsilon_{2}^{G} \cdot K_{3}\right) \operatorname{Tr}\left(\epsilon_{1}^{G} \cdot \epsilon_{3}^{G}\right)+2\left(K_{2} \cdot \epsilon_{1}^{G} \cdot \epsilon_{2}^{G} \cdot \epsilon_{3}^{G} \cdot K_{1}\right) \\
& \left.+2\left(K_{3} \cdot \epsilon_{2}^{G} \cdot \epsilon_{1}^{G} \cdot \epsilon_{3}^{G} \cdot K_{1}\right)+2\left(K_{2} \cdot \epsilon_{1}^{G} \cdot \epsilon_{3}^{G} \cdot \epsilon_{2}^{G} \cdot K_{3}\right)\right)+O\left(K^{4}\right)
\end{aligned}
$$

where $\mathrm{G}$ stands for graviton, dilaton or antisymmetric tensor and $\epsilon_{i}^{G}$ denotes their polarizations. 
- Three gauge bosons

$$
\left\langle V^{i} V^{j} V^{k}\right\rangle=\pi g_{c} \frac{i}{\sqrt{\alpha^{\prime}}} \epsilon^{i j k}\left[\left(\epsilon_{3}^{k} \cdot K_{1}\right)\left(\epsilon_{1}^{i} \cdot \epsilon_{2}^{j}\right)-\left(\epsilon_{2}^{j} \cdot K_{1}\right)\left(\epsilon_{1}^{i} \cdot \epsilon_{3}^{k}\right)+\left(\epsilon_{1}^{i} \cdot K_{2}\right)\left(\epsilon_{3}^{k} \cdot \epsilon_{2}^{j}\right)\right]
$$

A similar expression with $\epsilon^{i j k} \rightarrow \bar{\epsilon}^{l m n}$, etc. holds for the gauge bosons with $\mathrm{SU}(2)_{R}$ indices.

- Three scalars

$$
\left\langle V^{i l} V^{j m} V^{k n}\right\rangle=-\pi g_{c}^{\prime} \frac{2}{\alpha^{\prime}} \phi_{i l} \phi_{j m} \phi_{k n} \epsilon^{i j k} \bar{\epsilon}^{l m n}
$$

- One vector, two scalars

$$
\left\langle V^{i l} V^{j m} V^{k}\right\rangle=\pi g_{c}^{\prime} \frac{1}{\sqrt{\alpha^{\prime}}}\left(K_{1} \cdot \epsilon^{k}\right) \phi_{i l} \phi_{j m} \epsilon^{i j k} \delta^{l m}
$$

and similarly for an $\mathrm{SU}(2)_{R}$ vector $V^{n}$ with $\epsilon^{i j k} \rightarrow \epsilon^{l m n}, \delta^{l m} \rightarrow \delta^{i j}$.

- One graviton-two scalars:

$$
\left\langle V^{i l} V^{j m} V_{G}\right\rangle=\frac{\pi g_{c}^{\prime}}{2}\left(K_{1} \cdot \epsilon^{G} \cdot K_{1}\right) \phi_{i l} \phi_{j m} \delta^{i j} \delta^{l m}
$$

- One graviton/antisymmetric tensor-two vectors

$$
\begin{aligned}
\left\langle V^{i} V^{j} V_{G}\right\rangle=\frac{\pi g_{c}^{\prime}}{2} \delta^{i j}( & \left(K_{1} \cdot \epsilon^{G} \cdot K_{1}\right)\left(\epsilon_{1}^{i} \cdot \epsilon_{2}^{j}\right)-\left(K_{1} \cdot \epsilon^{G} \cdot \epsilon_{1}^{i}\right)\left(K_{1} \cdot \epsilon_{2}^{j}\right) \\
& \left.-\left(K_{2} \cdot \epsilon^{G} \cdot \epsilon_{2}^{j}\right)\left(K_{2} \cdot \epsilon_{1}^{i}\right)\right)
\end{aligned}
$$

and a similar expression for $\mathrm{SU}(2)_{R}$ vectors.

- Two vectors-one scalar

$$
\left\langle V^{i} \bar{V}^{l} V^{j m}\right\rangle=-\frac{\pi g_{c}^{\prime}}{2} \delta^{i j} \delta^{l m} \phi_{j m}\left(K_{2} \cdot \epsilon_{1}^{i}\right)\left(K_{1} \cdot \epsilon_{2}^{l}\right)
$$

coupling left and right sectors.

Away from self-dual radius there are other non-vanishing amplitudes. Namely

- Two vectors-one scalar

$$
\left\langle V^{\prime i} V^{\prime j} V^{33}\right\rangle=\pi g_{c}^{\prime} \frac{1}{4} m_{+} m_{-} \epsilon_{1}^{\prime i} \cdot \epsilon_{2}^{\prime j} \phi_{33}
$$

- Three-point coupling of left and right vectors

$$
\begin{aligned}
& \left\langle V_{L}^{+} V_{L}^{-} V_{R}^{3}\right\rangle=\pi g_{c}^{\prime}\left(\frac{m_{-}}{4}\right)\left(\epsilon_{1+}^{\prime} \cdot \epsilon_{2-}^{\prime}\right)\left(K_{1} \cdot \epsilon_{3}\right) \\
& \left\langle V^{+} V^{-} V_{L}^{3}\right\rangle=\pi g_{c}^{\prime}\left(\frac{m_{-}}{4}\right)\left(\epsilon_{1+}^{\prime} \cdot \epsilon_{2-}^{\prime}\right)\left(K_{1} \cdot \epsilon_{3}\right)
\end{aligned}
$$

- Three scalars

$$
\begin{aligned}
\left\langle V^{i l} V^{j m} V^{33}\right\rangle & =\pi g_{c} \frac{1}{2 R} \phi_{33} \phi_{i l} \phi_{j m} \\
\left\langle V^{i l} V^{j m} V^{33}\right\rangle & =-\pi g_{c} \frac{1}{2 \tilde{R}} \phi_{33} \phi_{i l} \phi_{j m}
\end{aligned}
$$


Open Access. This article is distributed under the terms of the Creative Commons Attribution License (CC-BY 4.0), which permits any use, distribution and reproduction in any medium, provided the original author(s) and source are credited.

\section{References}

[1] N. Hitchin, Generalized Calabi-Yau manifolds, Quart. J. Math. 54 (2003) 281 [math/0209099] [INSPIRE].

[2] M. Gualtieri, Generalized complex geometry, D.Phil. thesis, Oxford University, Oxford U.K. (2004) [math/0401221] [INSPIRE].

[3] C. Hull and B. Zwiebach, Double field theory, JHEP 09 (2009) 099 [arXiv:0904.4664] [INSPIRE].

[4] O. Hohm, C. Hull and B. Zwiebach, Background independent action for double field theory, JHEP 07 (2010) 016 [arXiv: 1003.5027] [INSPIRE].

[5] O. Hohm, C. Hull and B. Zwiebach, Generalized metric formulation of double field theory, JHEP 08 (2010) 008 [arXiv: 1006.4823] [INSPIRE].

[6] A.A. Tseytlin, Duality symmetric formulation of string world sheet dynamics, Phys. Lett. B 242 (1990) 163 [INSPIRE].

[7] A.A. Tseytlin, Duality symmetric closed string theory and interacting chiral scalars, Nucl. Phys. B 350 (1991) 395 [inSPIRE].

[8] W. Siegel, Superspace duality in low-energy superstrings, Phys. Rev. D 48 (1993) 2826 [hep-th/9305073] [INSPIRE].

[9] W. Siegel, Two vierbein formalism for string inspired axionic gravity, Phys. Rev. D 47 (1993) 5453 [hep-th/9302036] [INSPIRE].

[10] G. Aldazabal, D. Marques and C. Núñez, Double field theory: a pedagogical review, Class. Quant. Grav. 30 (2013) 163001 [arXiv:1305.1907] [INSPIRE].

[11] D.S. Berman and D.C. Thompson, Duality symmetric string and M-theory, Phys. Rept. 566 (2014) 1 [arXiv:1306.2643] [InSPIRE].

[12] O. Hohm, D. Lüst and B. Zwiebach, The spacetime of double field theory: review, remarks and outlook, Fortsch. Phys. 61 (2013) 926 [arXiv:1309.2977] [INSPIRE].

[13] O. Hohm, W. Siegel and B. Zwiebach, Doubled $\alpha^{\prime}$-geometry, JHEP 02 (2014) 065 [arXiv: 1306.2970] [INSPIRE].

[14] O. Hohm and B. Zwiebach, Double field theory at order $\alpha^{\prime}$, JHEP 11 (2014) 075 [arXiv: 1407.3803] [INSPIRE].

[15] O.A. Bedoya, D. Marques and C. Núñez, Heterotic $\alpha^{\prime}$-corrections in double field theory, JHEP 12 (2014) 074 [arXiv: 1407.0365] [INSPIRE].

[16] D. Marques and C.A. Núñez, T-duality and $\alpha^{\prime}$-corrections, JHEP 10 (2015) 084 [arXiv: 1507.00652] [INSPIRE].

[17] A. Coimbra, R. Minasian, H. Triendl and D. Waldram, Generalised geometry for string corrections, JHEP 11 (2014) 160 [arXiv:1407.7542] [INSPIRE].

[18] J. Scherk and J.H. Schwarz, How to get masses from extra dimensions, Nucl. Phys. B 153 (1979) 61 [inSPIRE]. 
[19] G. Aldazabal, W. Baron, D. Marques and C. Núnez, The effective action of double field theory, JHEP 11 (2011) 052 [Erratum ibid. 11 (2011) 109] [arXiv:1109.0290] [INSPIRE].

[20] D. Geissbuhler, Double field theory and $N=4$ gauged supergravity, JHEP 11 (2011) 116 [arXiv:1109.4280] [INSPIRE].

[21] M.B. Green, J.H. Schwarz and E. Witten, Superstring theory. Vol. 1: introduction, Cambridge Monogr. Math. Phys., Cambridge University Press, Cambridge U.K. (1988).

[22] J. Polchinski, String theory vol. I: an introduction to the bosonic string, Cambridge University Press, Cambridge U.K. (1998).

[23] D.J. Gross, J.A. Harvey, E.J. Martinec and R. Rohm, Heterotic string theory. 2. The interacting heterotic string, Nucl. Phys. B 267 (1986) 75 [InSPIRE].

[24] A. Giveon, M. Porrati and E. Rabinovici, Target space duality in string theory, Phys. Rept. 244 (1994) 77 [hep-th/9401139] [INSPIRE].

[25] J. Maharana, Scattering of stringy states in compactified closed bosonic string, Nucl. Phys. B 896 (2015) 657 [arXiv:1412.3888] [INSPIRE].

[26] M. Graña, R. Minasian, M. Petrini and D. Waldram, T-duality, generalized geometry and non-geometric backgrounds, JHEP 04 (2009) 075 [arXiv:0807.4527] [INSPIRE].

[27] D. Geissbuhler, D. Marques, C. Núñez and V. Penas, Exploring double field theory, JHEP 06 (2013) 101 [arXiv: 1304.1472] [INSPIRE].

[28] K. Lee, C. Strickland-Constable and D. Waldram, Spheres, generalised parallelisability and consistent truncations, arXiv:1401.3360 [INSPIRE].

[29] M. Graña and D. Marques, Gauged double field theory, JHEP 04 (2012) 020 [arXiv: 1201.2924] [INSPIRE].

[30] O. Hohm and S.K. Kwak, Double field theory formulation of heterotic strings, JHEP 06 (2011) 096 [arXiv:1103.2136] [INSPIRE].

[31] A. Coimbra, C. Strickland-Constable and D. Waldram, Supergravity as generalised geometry I: type II theories, JHEP 11 (2011) 091 [arXiv:1107.1733] [INSPIRE].

[32] I. Jeon, K. Lee and J.-H. Park, Stringy differential geometry, beyond Riemann, Phys. Rev. D 84 (2011) 044022 [arXiv:1105.6294] [INSPIRE].

[33] G. Dibitetto, J.J. Fernandez-Melgarejo, D. Marques and D. Roest, Duality orbits of non-geometric fluxes, Fortsch. Phys. 60 (2012) 1123 [arXiv:1203.6562] [INSPIRE].

[34] K. Lee, C. Strickland-Constable and D. Waldram, New gaugings and non-geometry, arXiv: 1506.03457 [INSPIRE].

[35] G. Dall'Agata, G. Inverso and M. Trigiante, Evidence for a family of $\mathrm{SO}(8)$ gauged supergravity theories, Phys. Rev. Lett. 109 (2012) 201301 [arXiv:1209.0760] [inSPIRE].

[36] M. Graña, R. Minasian, H. Triendl and T. Van Riet, Quantization problem in Scherk-Schwarz compactifications, Phys. Rev. D 88 (2013) 085018 [arXiv: 1305.0785] [INSPIRE].

[37] K. Lee, C. Strickland-Constable and D. Waldram, Spheres, generalised parallelisability and consistent truncations, arXiv:1401.3360 [INSPIRE].

[38] M.B. Schulz, T-folds, doubled geometry and the SU(2) WZW model, JHEP 06 (2012) 158 [arXiv:1106.6291] [INSPIRE]. 
[39] P.d. Bosque, F. Hassler and D. Lüst, Flux formulation of DFT on group manifolds and generalized Scherk-Schwarz compactifications, JHEP 02 (2016) 039 [arXiv:1509.04176] [INSPIRE].

[40] S.F. Hassan and A. Sen, Marginal deformations of $W Z N W$ and coset models from $O(d, d)$ transformation, Nucl. Phys. B 405 (1993) 143 [hep-th/9210121] [INSPIRE].

[41] K. Lee, Towards weakly constrained double field theory, arXiv: 1509.06973 [INSPIRE].

[42] E. Witten, Non-Abelian bosonization in two-dimensions, Commun. Math. Phys. 92 (1984) 455 [INSPIRE].

[43] D. Nemeschansky and S. Yankielowicz, Critical dimension of string theories in curved space, Phys. Rev. Lett. 54 (1985) 620 [Erratum ibid. 54 (1985) 1736] [INSPIRE].

[44] R.I. Nepomechie, Non-Abelian symmetries from higher dimensions in string theories, Phys. Rev. D 33 (1986) 3670 [Erratum ibid. D 36 (1987) 3290] [INSPIRE]. 